\title{
Die neue AO-CMF-Traumaklassifikation für Erwachsene - Überblick, Präzisions-Level und anatomische Module für Unterkiefer, Kiefergelenkfortsätze, Mittelgesicht und Orbita
}

\author{
Carl-Peter Cornelius, Laurent Audigé, Christoph Kunz, Carlos Buitrago-Téllez, \\ Andreas Neff, Michael Rasse, Joachim Prein
}

\section{Zusammenfassung}

Neue Bildgebungstechnologien haben Einzug in klinische Routineanwendungen gehalten und zu innovativen Behandlungskonzepten geführt, was v.a. bei Kiefergelenkfortsatzfrakturen und Verletzungen des Orbitarahmens sowie der internen Orbita (Orbitawände) zum Ausdruck kommt. Das neue AO-CMFTrauma-Klassifikationssystem für Erwachsene (3. Generation) beabsichtigt, den Fortschritten in der Frakturdarstellung auf CT-Basis Rechnung zu tragen. Drei Präzisionslevel bieten die Möglichkeit, Frakturlinienverläufe und -muster nach ihrer Lokalisation und hinsichtlich morphologischer Eigenschaften abgestuft bis zu einer bisher nicht dagewesenen Genauigkeit zu dokumentieren. Zur Dokumentation wird die CMF-Komponente des AOCOIAC(AO COmprehensive Injury Automatic Classifer-)Software-Programms verwendet. AOCOIAC CMF ist benutzerfreundlich strukturiert mit Visualisierung der Regionen und Subregionen innerhalb der anatomischen Module. Durch Anklicken wird die Topografie und Ausdehnung einer Fraktur in den Illustrationen markiert. Über Pulldown-Menüs sind die Eigenschaften der Frakturen ebenso anwählbar wie über Datentabellen mit ankreuzbaren Kästchen.

In Level 2 wird der Unterkiefer in 9 Subregionen unterteilt: Symphysis $(\mathbf{S})+\mathrm{Bi}-$ lateral: Body (B), Angle/Ascending Ramus (A), Coronoid Process (C), Condylar Process (P). Midface Level 2 differenziert laterale (= Zygoma/Zygomatic Arch Ensemble) und zentrale Kompartimente, wobei das zentrale Mittelgesicht 3 Etagen umfasst: Upper Central Midface (UCM), Intermediate Cen- tral Midface (ICM) und Lower Central Midface (LCM). In Level 3 werden die Subregionen im Processus condylaris und im Mittelgesicht in noch feinere Einheiten aufgegliedert. Zudem sind individuelle Patientenmerkmale, nämlich der prätraumatische Zahnstatus und die Alveolarfortsatzatrophie bei Zahnlosigkeit registrierbar. Auf dieser Basis wird es dann einfach, typische Frakturentitäten, d.h. Unterkiefer-Gelenkfortsatzfrakturen, Nasenskelett und Naso-Orbit-Ethmoidalfrakturen, Jochbein/Jochbogenfrakturen und Frakturen der Orbitawände in ihrem ganzen Variantenreichtum und bis ins Detail aufzuzeichnen, sowie Zahnhartsubstanz-, Zahnhalteapparatverletzungen und Alveolarfortsatzfrakturen zu dokumentieren. Die Morphologieeigenschaften der Frakturen (Fragmentation, Displacement/Dislokation, Knochendefekte) lassen sich als Attribute hinzufügen, um die Klassifikation auf dieser Stufe zu vervollständigen. In der Hoffnung der Autoren wird die vorliegende AO-CMF-Traumaklassifikation ein Nukleus zu einer verbesserten Standardisierung in der Befunderhebung und Diagnose von Traumen des gesamten Schädelskeletts, damit in $\mathrm{Zu}$ kunft gleiche Worte auch das Gleiche meinen.

The New Comprehensive AO CMF Trauma Classification for Adults Overview, Precision Levels and Anatomic Modules for the Mandible, Condylar Processes, Midface and Orbit

New imaging technologies are now reaching clinical practice and generating innovative treatment concepts that have become particularly apparent in fractures of the mandibular condylar process and the internal orbit. The new comprehensive AO CMF trauma classification for adults has become a reality, i.e., the 3rd generation, and is intended to reflect the progress in the delineation of fracture patterns using CT imaging. Three precision levels, from elementary through basic to focused, provide the opportunity to document the location and morphology of fracture lines and patterns in hitherto unprecedented detail and clarity. The CMF partition of the AOCOIAC (AO COmprehensive Injury Automatic Classifer) software programme for the entire human skeleton is used for this documentation. This is organised in a user friendly format visualising the regions and subregions within each anatomic module. The topographical extent of a fracture is conveniently marked by locating and klicking the mouse cursor on the illustrations. The properties of the fracture are selected in pull-down menus or by ticking checkboxes in a side table data sheet. In Level 2 the mandible is divided into 9 subregions: symphysis (S) + bilaterally: body (B), angle/ascending ramus (A), coronoid process $(\mathbf{C})$, condylar process $(\mathbf{P})$. Midface level 2 distinguishes between lateral (= zygoma/zygomatic arch ensemble) and central compartments, the latter being a stack of three horizontal partitions: upper central midface (UCM), intermediate central midface (ICM) and lower central midface (LCM). In level 3 the topography of the subregions is further subdivided to display even more refined areas for fracture delineation. Moreover, individual patient features, namely the pre-injury dental state and the degree of alveolar atrophy in edentulism can be recorded. Based on 
the refined structural layout, it is easy to plot common fracture entities, i.e. mandibular condylar process fractures, nasal and naso-orbito-ethmoid (NOE) fractures, zygoma/zygomatic arch fractures and fractures of the internal orbits in their enormous range of variability and with ultimate sophistication as well as to assess tooth injuries, periodontal trauma and fracture involvement of the alveolar process. As a key attribute the focused level 3 approach permits to document the fracture morphology in terms of fragmentation, displacement and bone loss. The authors wish to express their hope that the present AO CMF Trauma Classification will provoke a flagship initiative for an incorporation of future innovations and amendments in the standardised description and diagnosis of CMF trauma in order to give an identical meaning to seemingly common terminology.

\section{Einleitung - Definition und Zielset- zung von Klassifikationssystemen}

Einteilungen und Klassifikationssysteme sind allgegenwärtig. Ohne uns dessen immer im Einzelnen bewusst zu sein, wachsen wir wie selbstverständlich damit auf - z.B. in Kindergarten und Schule (Klassenprimus bis Schlusslicht?), oder bei Sport und Spiel (Bundesliga, Karten und Quartettspiele) - und gehen mit ihnen um - z. B. bei Bahnfahrten oder Flugreservierungen, Restaurants, Hotels oder auch bei der Bewertung und Auswahl von Produkten während Einkäufen oder im medizinischen Arbeitsalltag z.B. Essbarkeit (ja/nein) oder Güteklassen von Nahrungsmitteln (Obst, Gemüse Hühnereier usw.) bzw. Unterscheidung von Krankheitsentitäten (ICD), Differenzialdiagnose oder auch TNM-Tumorklassifikation mit Grading und Staging.

In Biowissenschaften und Medizin werden neuartige Methoden und Techniken in Diagnostik oder Therapie ganz regelmäßig im Zusammenhang mit präzisierten und überarbeiteten Kategorien oder auch als Updates früherer Systematisierungen und Einteilungsvarianten publiziert.

Hinter dem Vokabular vieler Vortragstitel und Artikelüberschriften stecken eigentlich Klassifikationen und Klassifikationsprozesse sowie in Abhängigkeit davon häufig Entscheidungskriterien: Gruppierung, Code und Codierung, Typisierung, Rating, Schweregrad, Scale, Score oder Severity Index sind typische Beispiele.

Die Begriffe Klassifikation und Klassifizierung haben etwa folgende allgemeingültige Definition: Eine Klassifikation, Typifikation oder Systematik entspricht einem systematischen Arrangement oder einer Einteilung z.B. von Lebewesen, Objekten, Konstellationen oder Phänomenen in Gruppen, Typen oder Kategorien, die zur Einordnung oder gegenseitigen Abgrenzung verwendet werden.
Die Kategorien oder Klassen entstehen durch Klassifizierung, d.h. sie werden auf der Basis ähnlicher und/oder gegensätzlicher Merkmale nach einem Plan oder durch eine Abfolge logischer Schritte konzipiert. Eine Klassifizierung beruht auf den Grundlagen von Begriffsbildung, Abstraktion und induktivem Denken.

Die Auswahl einer passenden Klasse innerhalb einer gegebenen Klassifikation für ein Item oder einen Sachverhalt wird als Klassierung oder Klassenzuordnung bezeichnet.

Klassifikationen haben v.a. den Zweck, den Umgang mit großen Mengen an Primärinformationen oder mit einer Vielzahl von Dingen zu vereinfachen, indem besondere Aspekte betont und nicht essenzielle Eigenschaften und Details eliminiert werden. Größere Kategorien werden so oft in immer kleinere Subkategorien gegliedert, bis in einer Gruppe keine gravierenden Unterschiede mehr vorhanden sind (Cornelius et al. 2012 in AO CMF Manual).

In Analogie dazu versteht sich eine Klassifikation in der Traumatologie als eine Sammlung möglicher Klassen, in welche die Grundgesamtheit von Fraktur- bzw. Verletzungsmustern nach bestimmten Vorgaben bzw. einem strukturierten Modus einsortiert werden.

Im Unterschied zu einer Klassifikation bzw. Klassierung dient ein „Fracture Mapping“ der exakten Beschreibung von Frakturlinienverläufen bzw. eines Frakturmusters, das ein Individuum erlitten hat, bis hin zu kleinsten Einzelheiten, wobei die Gesamtkonfiguration und Detailgenauigkeit des Mappings letztlich die Grundlage einer patientengerechten Behandlung darstellt. Demgegenüber bleibt eine Klassierung auf einem früheren Niveau stehen mit dem Ziel, die Menge an Informationen zu reduzieren und zugleich problemorientiert zu bleiben, ohne aber wegweisende Parameter auszulassen.
Jede Klasse umfasst identische Eigenschaften. Genauso wie Gruppen und Subgruppen sind Klassen jedoch disjunktiv, d.h. sie schließen sich nach bestimmten Kriterien oder Charakteristika gegenseitig aus. Das bedeutet jedoch keineswegs zwangsläufig, dass es nicht auch Übereinstimmungen oder Überlappungen für andere Merkmale geben kann (Abb. 1).

Es ist ein grundsätzliches Problem, solche Ausschlusskriterien bei der Neukonzeption eines Klassifikationssystems vorab festzulegen. Methodisch macht es insofern Sinn, ein Klassifikationsmodell stufenweise in einem mehrphasigen iterativen Prozess zu entwickeln und durch wiederholte Überprüfung von ersten Vorschlägen über Pilotstudien und klinische Multicenterstudien zu validieren, bevor das resultierende Modell schließlich für prospektive klinische OutcomeUntersuchungen einer oder unterschiedlicher Therapievarianten eingesetzt werden kann (Audigé et al. 2005) (Abb. 2).

Soweit das intellektuell überhaupt $\mathrm{zu}$ leisten ist, sollten Erfahrungswerte aus bekannten Behandlungsverfahren bei der Formulierung der Erstversion eines Klassifikationsvorschlags ausgeklammert werden. Stattdessen sollten sich die Vorschläge in den frühen Entwicklungsphasen ausschließlich auf das anatomische Substrat von Frakturmustern, sprich auf die Lokalisation und Morphologie einer Fraktur, beziehen.

Schon in der Anfangsphase auf „Therapierelevanz" ausgerichtete Klassifizierungsvorschläge führen $\mathrm{zu}$ kognitiven Verzerrungen („Bias“) und de facto ins Aus, wenn trotz immanenter Präjudizierung eine rationale Basis für prospektive Therapiestudien geschaffen werden soll.

Angesichts des hohen Aufwands und der Schwierigkeiten, die mit der Erstellung einer validen Traumaklassifikation verbunden sind, ist von einigen Fachvertretern immer wieder das Mantra zu hören, 

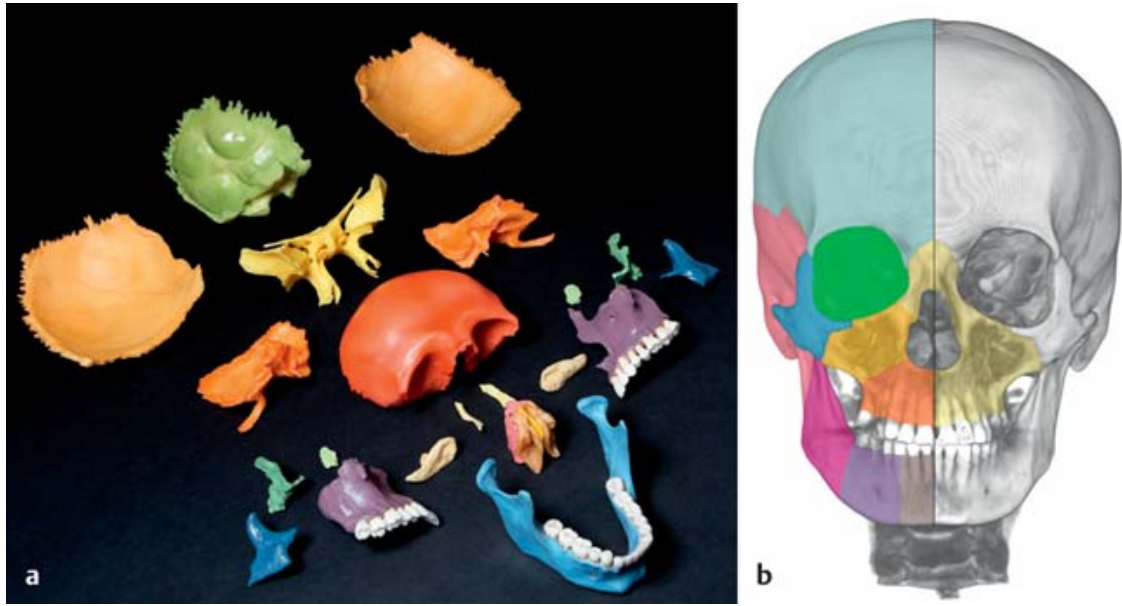

Abb. $1 \mathbf{a}$ und $\mathbf{b}$ a Sprengschädel mit kolorierten Einzelknochen (es fehlen: Vomer und Gehörknöchelchenketten). b 3-D-Darstellung des Schädelskeletts mit kolorierten Regionen und Subregionen. Mögliche Parameter für eine CMF-Traumaklassifikation wären z. B. 1. die Zugehörigkeit der Knochen zum Gesichtskelett, zur Schädelbasis oder zum Schädeldach, wobei sich dabei erste Überlappungen ergeben; 2. die Lokalisation der Knochen nach Etagen (Mandibula, bzw. Untergesicht, Mittelgesicht, kraniofazialer Übergang bzw. Schädelbasis, Schädeldach) oder in zentrallateraler Orientierung. Die Farbgebung der Knochen ist frei wählbar und entspricht nicht der Realität, sie ist daher zur Frakturklassierung gänzlich unbrauchbar und kann lediglich didaktische Hilfen geben. Grundsatzproblem: Die Frakturlinienverläufe halten sich nicht an die Suturen oder Umrisse der gezeigten Einzelknochen, sondern involvieren und durchsetzen diese in unterschiedlichsten Mustern. Jeder Knochen bzw. jede Sklelettregion muss deshalb in entsprechende Untereinheiten oder Subregionen aufgeteilt werden.

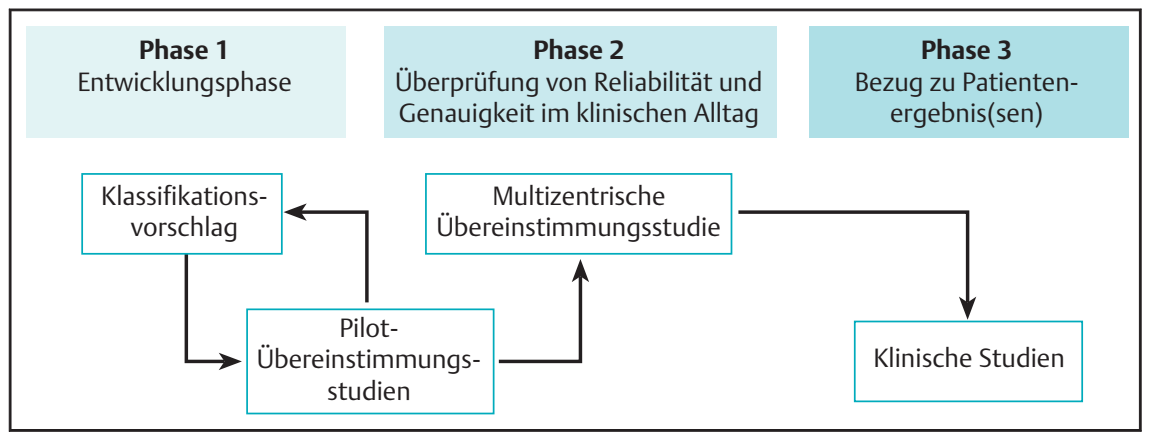

Abb. 2 Methodische Entwicklung und Validierung einer Klassifikation in einem 3-Phasen-Prozess (mit freundlicher Genehmigung der AO Foundation, Davos [15]). In Phase 1 werden der Klassifikationsvorschlag und die Pilot-Übereinstimmungsstudien nach Durchsicht und Dokumentation von CT-Serien (von bis zu 150 Patienten) durch mehrere Auswerter in einer Wiederholungsschleife solange diskutiert und optimiert, bis die Reproduzierbarkeit und Übereinstimmung der Ergebnisse akzeptable Werte erreicht hat. In Phase 2 geht dieser Vorschlag dann innerhalb einer Multicenterstudie im klinischen Alltag auf den Prüfstand. In Phase 3 wird die validierte Klassifikation dann zu klinischen Behandlungsstudien verwendet und kann erst dann abschließend nach ihrer potenziellen Therapierelevanz modifiziert werden, um zukünftig Entscheidungsgrundlagen liefern zu können.

man brauche gar keine Klassifikationen und bekomme die traumatologische Patientenversorgung auch so in den Griff. Letzteres mag durchaus zutreffen. Gegen ersteres spricht die allgemeine Lebenserfahrung, denn alle Werte und Bewertungssysteme sowie jegliche Begriffsbildung stehen in Wechselbeziehung zu Klassifizierungen und sind die Grundlage jeder Verständigung. Auch die Beurteilung einer Fraktur oder Verletzung und die Entscheidungsfindung zu einem individualisierten Behandlungsmodus macht davon keine Ausnahme.

Voraussetzung für eine universelle, weltweite Verständigung (Kommunikation und Zusammenarbeit) in traumatologischen Fragen ist ein konsistentes und reproduzierbares Klassifikationssystem. Nur damit bekommt eine Diagnose, sei es in Form eines Codes, eines Indexes oder eines Icons, allseits die gleiche standardisierte Bedeutung, sodass eine Dokumentation und ein Austausch „har- ter Daten“ (z. B. zur Qualitätskontrolle, Benchmarking oder innerhalb von Vergleichsstudien zwischen mehreren Institutionen) über elektronische Medien stattfinden kann.

Die neue AO-CMF-Traumaklassifikation für Erwachsene ist zur Analyse und Dokumentation von Frakturen im Bereich des gesamten Schädelskeletts auf der Basis von CT-Daten konzipiert. Kernstück ist ein visuell orientiertes Software-Programm, das neben Illustrationen und Icons zur Lokalisation der Frakturen Pull-down-Menüs bereithält, um die Morphologie der Frakturen näher zu charakterisieren. Außer der visuellen Darstellung wird für jedes Frakturmuster in den jeweils als anatomisches Modul zusammengefassten Regionen Unterkiefer, Kiefergelenkfortsätze, Mittelgesicht, Orbitae und Schädelbais ein alphanumerischer Code generiert.

Die CMF Software Application in der Version 4.0 ist als Komponente in die AO Comprehensive Injury Automatic Classifier (AOCOIAC) Software implementiert, die auch die Müller-AO-Klassifikation (Müller et al. 1990) und die AO Pediatric Comprehensive Fracture Classification of long Bones (Slongo et al. 2007) beinhaltet.

Das Akronym COIAC wurde seinerzeit in Anlehnung an die in den 70er-Jahren populäre US Fernsehkrimiserie „Kojak (Einsatz in Manhattan)" ersonnen. Vermutlich mit der Absicht, wohltuende Assoziationen mit dem glatzköpfigen New Yorker Detektiv wachzurufen, der mit stets großer Gelassenheit, professionellem Zynismus, coolen Sprüchen („Entzückend, Baby“ - „Who loves you baby“ oder „Is es denn wahr?“) sowie als Markenzeichen einen Lollipop lutschend auch die vertraktesten Fälle zu lösen wusste.

Im Folgenden werden die Features der neuen bzw. 3. Generation AO CMF Trauma Classification in ihren Grundzügen vorgestellt. Die aus AOCOIAC übernommenen Icons sind in englischer Sprache belassen. Auch in den Abbildungslegenden und Begleittexten wird Englisch benutzt - falls als notwendig erachtet, mit lateinischer und deutscher Übersetzung in Klammern oder nach Schrägstrichen. 


\section{Kurze Historie der CMF-Traumaklassifikationen}

Bis heute werden Mitttelgesichtsfrakturen, die mit einer Okklusionsstörung einhergehen, weltweit als Le-Fort-Frakturen bezeichnet. Die einfache Unterscheidung von 3 Le-Fort-Frakturtypen ist der Prototyp eines erfolgreichen Klassifikationssystems für Gesichtsschädelverletzungen, um nicht zu sagen der „Klassiker"1 aller Klassifikationen. Die experimentellen Studien von René Le Fort (1869-1951) reichen an den Anfang des letzten Jahrhunderts (1901) zurück (Tessier 1972a und b). An insgesamt 35 Schädeln, auf die mehrfach mit einem Holzblock (ohne dass es Belege dafür gibt, wird gelegentlich kolportiert, es sei das Standbein eines Konzertflügels gewesen) eingeschlagen wurde oder die man an einer Schlaufe aufgehängt wie ein Kugelstoßpendel gegen eine abgerundete Tischkante schwingen ließ, konnte Le Fort die Schwach- bzw. „Sollbruchstellen“ in der von mehreren Kavitäten durchsetzten Wabenkonstruktion des Schädels identifizieren. Die entsprechenden Bruchlinien verlaufen bekanntlich auf 3 Ebenen: im einfachsten Fall der horizontal angeordneten Le-Fort-I-Fraktur geringfügig oberhalb des Gaumendachs bzw. Nasenbodens auf einer Transversalebene durch beide Maxillae; bei einer Le-Fort-II- oder Pyramidalfraktur entlang der seitlichen Suturen beider Maxillae und ihrer nasofrontalen Fortsätze bis in den Bereich der Nasenwurzeln; und bei Le-Fort-III-Frakturen im Bereich der Suturen zwischen Viszeround Neurocranium (zygomatico-frontal, zygomatico-sphenoidal, ethmoido-, lacrimo- und naso-frontal).

Definitionsgemäß sind Le-Fort-Frakturen bilateral lokalisiert und schließen immer die Processus pterygoidei ein. Nach heutiger Auffassung handelt es sich um Low-Energy-Verletzungsmuster, im Gegensatz zu Hoch-Energie-Dezelerations- oder Aufpralltraumen mit multilokulär ausgedehnten Frakturlinienverläufen und pan-cranio-fazialer Multifragmentierung, die sich nicht an die

\footnotetext{
1 Die Ähnlichkeit der Begriffe provoziert zu Wortspielen. Die genaue Bedeutung der Worte ist deshalb interessant: Klassik von lat. „classicus“ = zum oberen Bürgerrang gehörend - klassisch $=$ in tradierter mustergültiger Weise ausgeführt und daher als Maßstab geltend. Umgangssprachlich: „Das ist ja Klasse“. - Allen Begriffen liegt eine Klassierung zugrunde (zur Bedeutung der Epoche Klassik siehe auch Schmalzriedt 1971).
}

Rahmenkonstruktion des Gesichtskeletts halten (Manson 1986).

Weil die Le-Fort-Klassifikation nur ein erstes kursorisches Raster liefert, wurde sie im Laufe des letzten Jahrhunderts vielfach ergänzt, um Details und spezielle Entitäten genauer beschreiben zu können:

- Mittelgesicht (Wassmund 1927, Donat et al. 1998),

- Jochbein -/Zygoma (Zingg et al. 1992),

- orbito-zygomatische und Orbito-ethmoidal Region (Jackson 1989),

- Naso-Orbito-Ethmoidal (NOE) Region (Gruss et al. 1985, Markowitz et al. 1991),

- Orbita (Hammer 1995, Carinici 2006, Jaquiéry et al. 2007),

- mediale Orbitawand (Nolasco and Mathog 1995),

- Gaumen (Chen et al. 2008),

- Mittelgesicht in Zusammenhang mit Schädelbasis (Buitrago-Téllez et al. 1999, 2002, Bächli et al. 2009),

- Frontobasis (Madhusdan et al. 2006),

- laterale Schädelbasis, Schläfenbein (Os temporale) (Rafferty et al. 2006),

- Unterkiefer (Mandibula) (Spiessl 1989, Roth et al. 2005, Buitrago-Téllez et al. 2008, u.v.m.; vgl. Cornelius et al. 2014),

- Kiefergelenkfortsatz (Spiessl und Schroll 1972, Loukota et al. 2005),

- panfaziale Verletzungen und Avulsionen (Clark et al. 1995).

Ferner wurden mehrere Vorschläge für Schweregrad bzw. Severity Scores bei CMF-Frakturen publiziert (Cooter und David 1989, Joos et al. 1999, Bagheri et al. 2006, Zhang et al. 2006).

\section{Entwicklung einer modernen AO-CMF-Frakturenklassifikation}

Auf Grundlage der Habilitationsschrift von C.H. Buitrago-Téllez (1998) wurde in einem AO-Projekt bis etwa zur Mitte des letzten Jahrzehnts die Idee verfolgt, eine moderne, auf dem sog. Tripartitionskonzept beruhende CMF-Klassifikation zu entwickeln. Die Tripartition wurde seinerzeit in der „Comprehensive Classification of Long Bones" von Maurice Müller verwendet, um für eine Region bzw. einen „Prinicipal bone“ durch 3-mal wiederholte Dreiteilung nach dem Vorbild einer hierarchischen Baum-AstZweig-Struktur eine Rangskala (3 Typen - 9 Gruppen - zuletzt: 27 Subgruppen) unterschiedlicher Schweregrade aufzustellen und in einem alphanumerischen Code zu dokumentieren.
Diese 1. Generation der AO CMF Classification basierte bereits auf CTBildgebung und unterschied 3 horizontale und 3 vertikale Mittelgesichtseinheiten. Dabei wurde einheitsübergreifend die anteriore und mittlere Schädelbasis in das Mittelgesicht einbezogen, um ein $3 \times 3 \times 3$-Schweregrad-Ranking herzustellen. Obwohl damit endlich die Absicht verwirklicht war, von der LeFort-Einteilung abweichende Frakturlinienverläufe genau dokumentieren $\mathrm{zu}$ können, erwies sich das Klassifikationssystem in der Praxis als schwierig zu verinnerlichen und als wenig tauglich für die tägliche Kommunikation.

Ein daraufhin zwischen 2005 und Ende 2007 von einer international besetzten Task-Force-Gruppe entwickeltes CMFTrauma-Klassifikationsmodell für das Mittelgesicht wurde mit großem Enthusiasmus so vielschichtig angelegt und ging so sehr ins Detail, dass damit mühelos ein Fracture Mapping durchführbar gewesen wäre. Die Subregionen im Mittelgesicht in dieser 2.Generation der AOCMF Trauma Classification glichen dabei einem kunstvoll zusammengesetzten Patchwork (Abb.3), entzogen sich bedauerlicherweise aber der Definition ihrer Lage und Grenzen mit anatomischen Termini.

Sowohl das Modell der 1. als auch der 2. Generation waren zu diffizil und unübersichtlich, um die 1 . Phase der methodischen Validierung (Abb. 2) zu bestehen.

\section{Aktuelle AO-CMF-Frakturen- Klassifikation}

Schon immer haben sich AO-TraumaKlassifikationen auf eine umfassende visuelle Darstellung mit detaillierten Illustrationen verlassen, um eine weitgehend nonverbale Kommunikation zu erreichen und zugleich möglichst intuitiv und selbsterklärend zu sein.

In der hier präsentierten 3. Generation der AO CMF Fracture Classification dominieren ebenfalls grafisch-optische Elemente und Icons, damit Engramme hinterlassen werden.

Überdies wurde eine Liste von Anforderungskriterien verwirklicht:

- minimalistisches Design- mit anatomischen Modulen (Unterkiefer, Kiefergelenkfortsätze, Mittelgesicht, Orbita, Schädelbasis, Schädeldach - Abb. 4) 


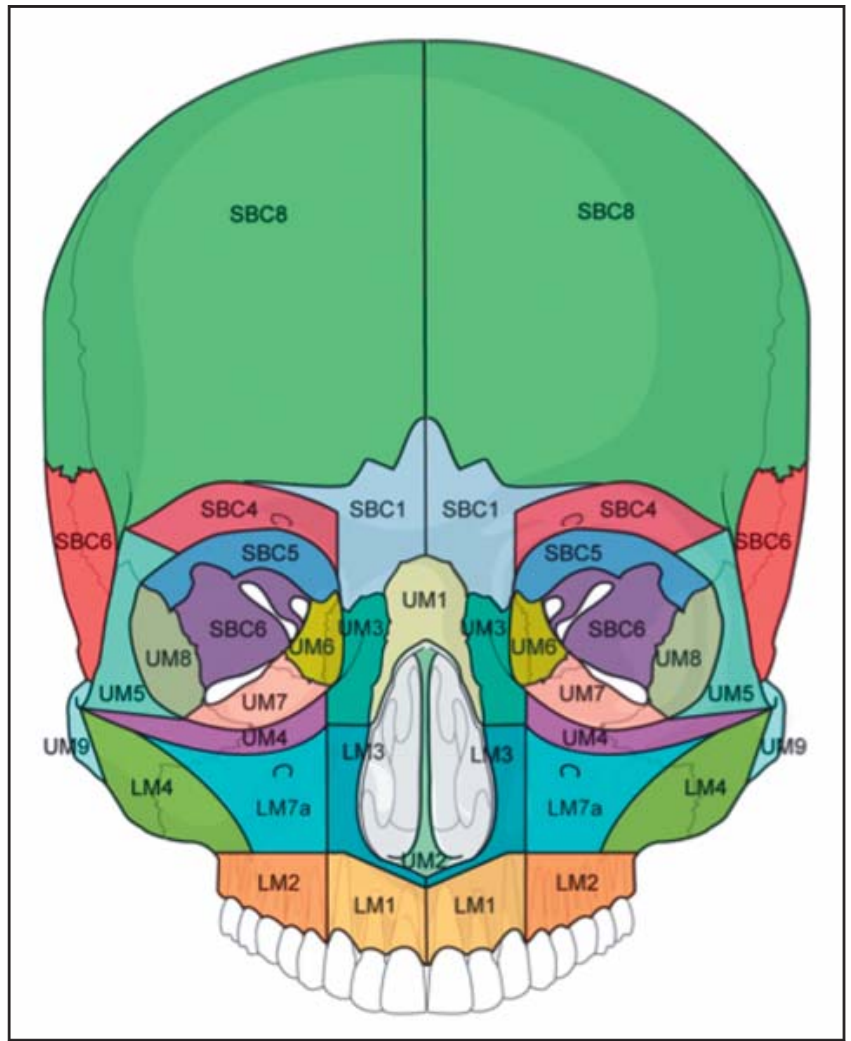

Abb. 3 Untereinheiten im Mittelgesicht und vorderen Schädeldach nach Darstellung der 2. Generation der AO CMF Fracture Classification.

- Präzisions-Level, mit denen sich verschiedene Ansprüche an Genauigkeit erfüllen lassen

- CT-Bildgebung als gemeinsame Basis für alle Module und Level

- topografische Beschreibung der Frakturen

- Implementierung der Le-Fort-Klassifikation in das Mittelgesichtsmodul

- Beschreibung der Frakturen-Morphologie (Fragmentation, Dislokation)

Die aktuelle CMF Fracture Classification erhielt im Juli 2012 das Approval vom AO CMF International Board (21st Meeting) und ist damit offiziell und formal die neue AO CMF Fracture Classification.

\section{Anatomische Module}

In der Müller-AO-Klassifikation werden folgende 4 „Principal Bones“ mit der angegebenen Nummerierung im Schädelskelett unterschieden:

- 91 Mandible

- 92 Midface

- 93 Skull Base

- 94 Cranial Vault

\section{Präzisions-Level}

DIe Wahl des sog. Präzisions-Levels bestimmt die Detailgenauigkeit, mit der

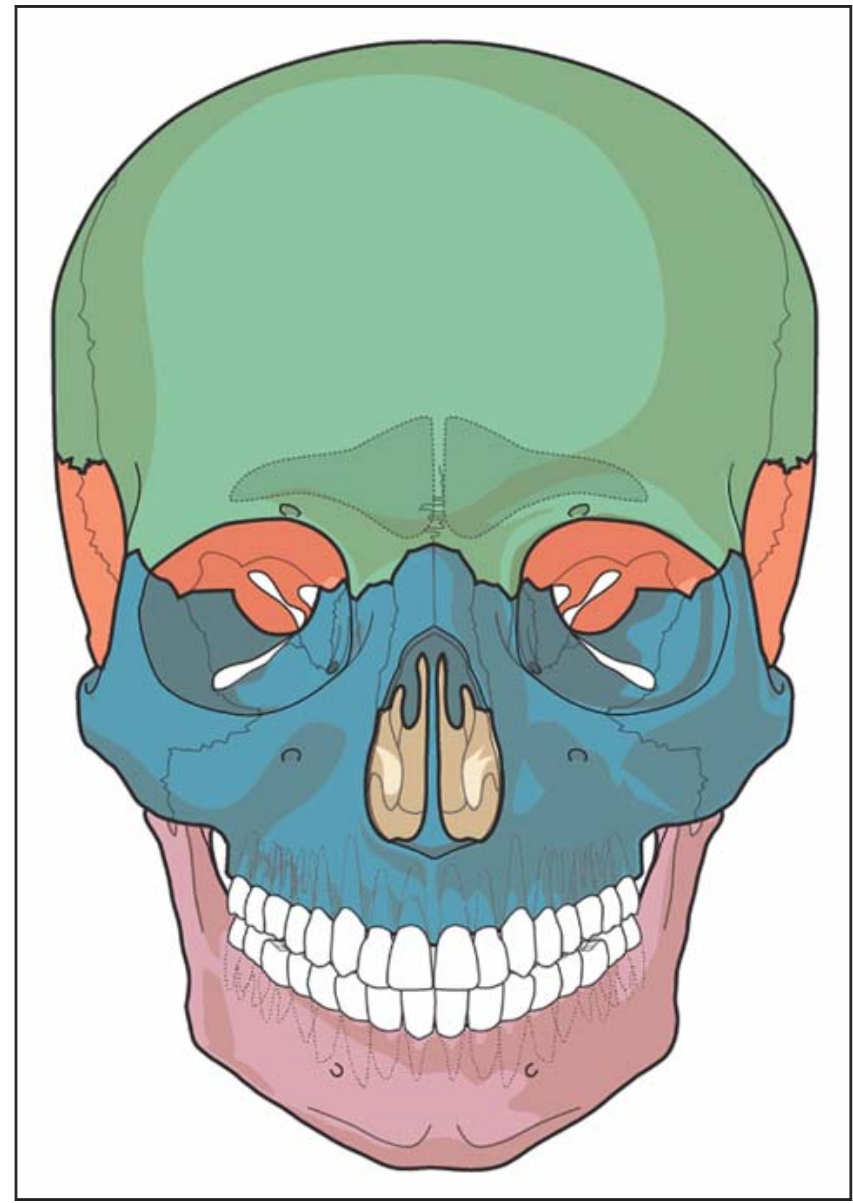

Abb. 4 „Principal Bones“ aus der AO-Müller-Klassifikation (mit freundlicher Genehmigung der AO Foundation, Davos [15]).

Frakturlinien oder ein Frakturmuster klassifiziert und dokumentiert werden.

Die aktuelle AO-CMF-Frakturenklassifikation weist zurzeit 3 Genauigkeitsstufen auf:

- Level 1 Elementary System

- Level 2 Basic regional System

- Level 3 Focused subregional System

Der Level 1 ist die grundlegende Variante der AO-CMF-Frakturenklassifikation. Gewissermaßen als kleinster gemeinsamer Nenner besitzt der Level 1 nur 4 Klassen, die nichts anderem entsprechen als den 4 „Principal Bones“ bzw. den Hauptregionen des Schädelskeletts der Müller-AO-Klassifikation.

Im Level 2 lässt sich die Topografie der Frakturen innerhalb der 4 anatomischen Elementarmodule exakter angeben. Die anatomischen Grenzen der Regionen und Subregionen sind im Einzelnen definiert und in einer Serie zugehöriger Tutorials sowie einer umfangreichen Fallsammlung schriftlich fixiert (Audigé et al. 2014 a und b, Buitrago et al. 2014, Cor- nelius et al. 2014a bis d, Kunz et al. 2014a und b). Im Unterkiefer sind zur eindeutigen topografischen Zuordnung „grenzüberschreitender“ Frakturlinien außerdem sog. „Transitional Zones“ eingefügt.

Im Mittelgesicht wird ein zentrales Kompartiment von der lateralen Mittelgesichtsregion differenziert und die interne Orbita in 4 Wände und die Apexregion aufgeteilt.

Der Level 3 ermöglicht die Aufzeichnung individueller Befunde (Zahnstatus, Atrophiegrad der Alveolarfortsätze) und gestattet die Dokumentation der Frakturen Morphologie (im Unterkiefer: Fragmentation; im Oberkiefer: Fragmentation und Displacement).

Ein weiterer Fokus von Mandible Level 3 ist außerdem auf die exakte Erfassung von Kiefergelenkfortsatzfrakturen gerichtet. Im Midface Level 3 wird die Differenzierung der Subregionen ebenfalls noch differenzierter. NOE-Region, Zygoma, Orbitaränder und -wände werden 


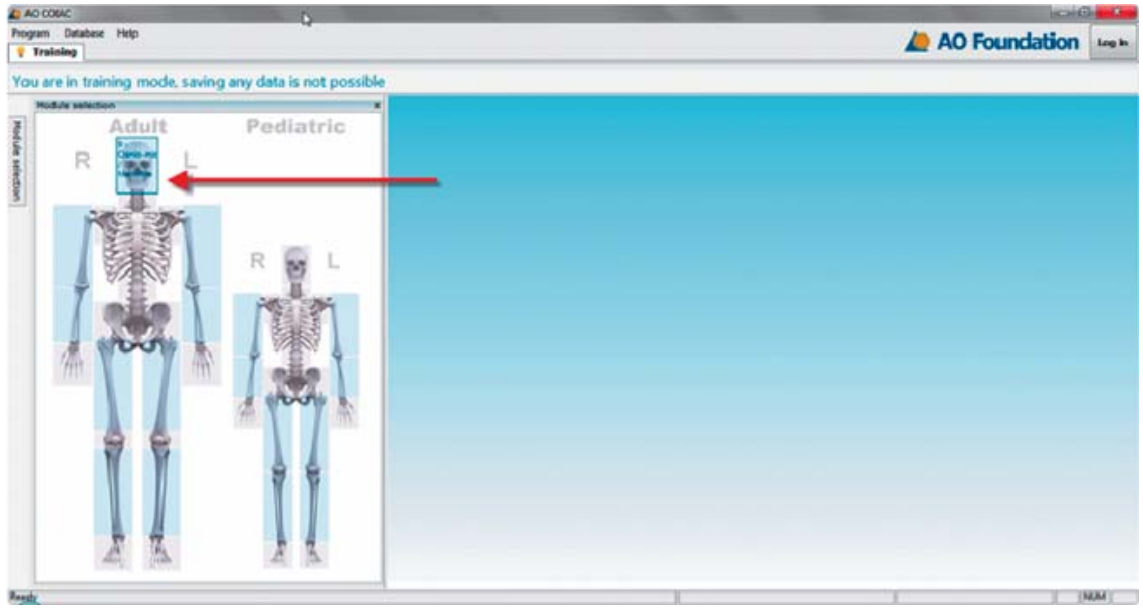

Abb. 5 AOCOIAC Start Window. Auswahl der anatomischen Module - CMF Adult.

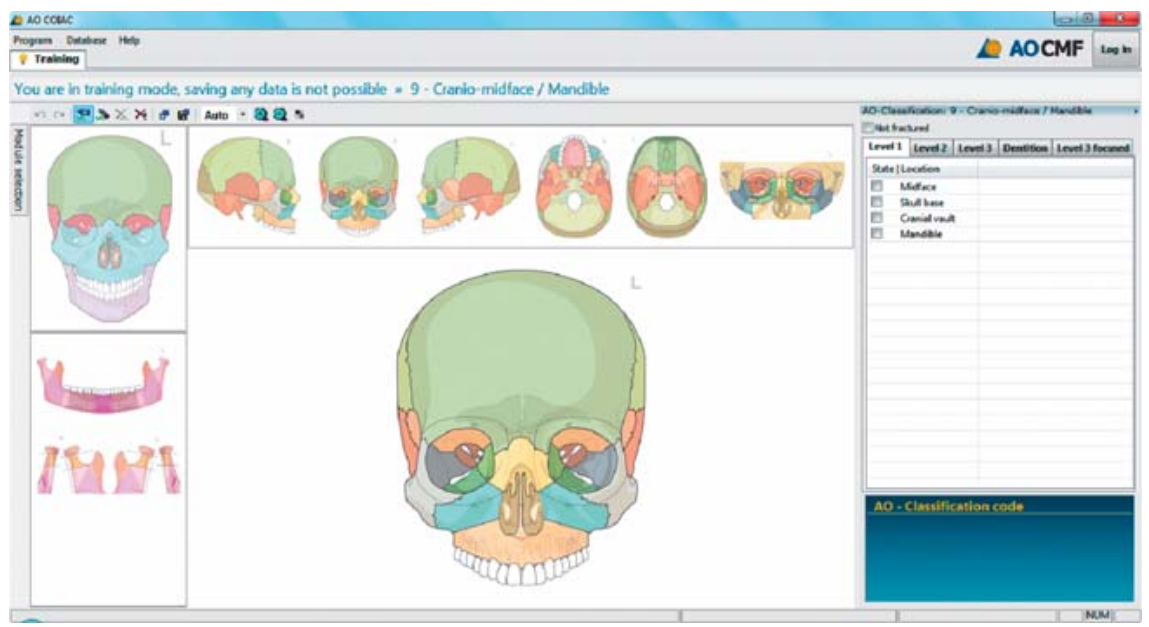

Abb. 6 Übersicht mit den Icons für alle 3 Präzisions-Level. Das angewählte Icon erscheint im zentralen unteren Fenster (s. Text).

in kleinere Bausteine aufgegliedert, was eine Abbildung auch von multifragmentären Frakturmustern bis in feine Details ermöglicht. Außerdem können individuelle Patientenbefunde (wahrscheinlicher Vor-Trauma-Zahnstatus und Alveolarkammatrophie) aufgezeichnet werden und demgemäß Zahn- und Parodontalverletzungen sowie Alveolarfortsatzfrakturen dokumentiert werden.

Bislang nimmt der Level 3 die höchste Präzisionsstufe ein. Bei Bedarf lassen sich für wissenschaftliche Zwecke weitere Level generieren, bspw. zur Erfassung von Weichgewebeverletzungen oder anderer klinischer Parameter.

\section{Start Window AOCOIAC}

Das AOCOIAC-Startfenster zeigt 2 menschliche Skelette (Abb.5), die Zugang zu sämtlichen erhältlichen anatomischen Modulen im Erwachsenenund Kindesalter bieten.
Das AO-CMF-Frakturen-KlassifikationsSystem für Erwachsene wird durch Anklicken des adulten Schädelskeletts aktiviert.

Die AO-CMF-Frakturenklassifikation kann im ausgewachsenen Schädelskelett, d.h. bei bleibender Dentition oder Zahnlosigkeit, verwendet werden. Bei der Befundung sollte das Trauma nicht länger als 10 Tage zurückdatieren.

Dann öffnet sich das AO-CMF-Frakturenklassifikationsfenster mit den Icons aller Präzisions-Level (Abb.6). Im Rahmen oben links ist das Schädelskelett für die Level-1-Klassifikation wiedergegeben.

Das Rechteck darunter präsentiert die Mandibula in Panoramaansicht für Level 2 sowie die Kiefergelenkfortsätze in Frontal- und Lateralansicht für Level 3.

Die obere Reihe zeigt die Icons für Mittelgesicht und Schädeldach in den Late- ralansichten (rechts und links) und von frontal - alle für Level 2, die Schädelbasis von extra- und intrakraniell für Level 2 und 3 und eine Frontalansicht der Orbita, Nasenskelett- und Jochbeinregion beidseits für Level 3.

Ein Mausklick auf das gewünschte Icon befördert es in Vergrößerung in das untere zentrale Fenster.

In der Seitentabelle rechts sind noch einmal alle Regionen, Subregionen und die 3 Präzisions-Level mit sämtlichen Features als Data Sheet mit ankreuzbaren Kästchen aufgeführt. Eingaben in diese Tabelle werden in die Illustrationen und Pulldown-Menüs umgesetzt und vice versa.

In der Box mit dunkelblauem Hintergrund (Fensterecke unten rechts) wird der alphanumerische Frakturencode jeder Frakturvariante angezeigt.

\section{Unterkieferfrakturen - 91 Mandible}

Mandible Level 2

Im Level 2 wird die Region Unterkiefer in 9 Subregionen eingeteilt: die Symphyse anterolateral, 2 Korpus-(Body-)Abschnitte auf jeder Seite lateral, kombinierte Kieferwinkel/aufsteigende AstUntereinheiten (Angle/ascending ramus) und die Muskel- (Coronoid Process) und Gelenkfortsätze (Condylar Process) (Abb. 7).

Der Panorama View des Unterkiefers wurde deshalb für AOCOIAC als Icon gewählt, weil Panoramaröntgenschichtaufnahmen die am weitesten verbreitete Screeningtechnik zur Erfassung pathologischer Prozesse oder Frakturen im Unterkiefer sind.

Die Seitengrenzen der zentral im anterioren Unterkieferbogen lokalisierten Symphyse werden durch die Wurzeln der Eckzähne (caninus) bestimmt. Insofern entspricht die im vorliegenden Kontext sog. Symphyse dem intercaninen Knochenabschnitt.

Die Bezeichnung Unterkieferkörper (Body) bezieht sich jeweils auf den Knochenabschnitt zwischen Eckzahn und innerem Kieferwinkel.

Kieferwinkel und aufsteigender Ast sind zur Angle/Ramus Subdivison mit einem irregulären 5-eckigen Umriss zusammengefasst. Die vordere vertikale Begrenzung dieses Fünfecks bewegt sich 
hinter dem 3. Molaren bzw. parallel zu dessen distaler Wurzel abwärts. Kaudal und hinten unten halten sich die Umrisslinien an die Ränder des äußeren Kieferwinkels. Nach oben läuft die Angle/Ramus Subdivision giebelförmig aus, wobei der Dachfirst auf den tiefsten Punkt der Incisura mandibulae (sigmoidea) fällt. Die Giebelseiten bilden die Basis für den Condylar bzw. Coronoid Process.

Außer diesen 9 Subregionen sind im Mandibularbogen 4 Übergangs- bzw. „Transitional Zones“ vorgegeben. Dabei handelt es sich um vertikal ausgerichtete Korridore in der Kronenbreite der Eckzähne bzw. der 3. Molaren. Die beiden Korridorpaare, d.h. die „Anterior“ bzw. „Posterior Transitional Zones“, haben die Funktion, Frakturlinien an Grenzen von 2 benachbarten Subregionen eindeutig zuzuordnen.

Verläuft eine Frakturlinie in voller Gänze innerhalb einer der „Transitional Zones“, so wird sie der dorsal davon lokalisierten Subregion zugeschrieben. Mit anderen Worten: komplett in der „Anterior Transitional Zone“ lokalisierte Frakturlinien werden dem Unterkieferkorpus, komplett in der „Posterior Transitional Zone“ befindliche Frakturen der Angle/Ramus Subdivision zugerechnet.

Frakturlinien, deren Ausläufer von dorsal bzw. ventral kommend in einer der Übergangszonen enden, werden der vorwiegenden Lokalisation entsprechend entweder der dorsal oder ventral gelegenen Subregion eingeordnet.

Falls Frakturen zu beiden Seiten über die „Transitional Zones“ hinausgehen, werden beide Subregionen einbezogen.

Im zahnlosen Kiefer (Level 3) sind die „Transitional Zones“ approximativ als $5 \mathrm{~mm}$ bzw. $7 \mathrm{~mm}$ breite vertikale Streifen vor dem Foramen mentale und in der Retromolarregion platziert.

Singuläre bzw. unilokuläre Frakturen oder Mehrfach- („Plural“) bzw. multilokuläre Frakturen (z.B. unilateral in benachbarten oder räumlich getrennten Regionen, bilateral) werden durch Kennzeichnung einer oder mehrerer Subregionen dokumentiert.

Statt die Subregionen mit Mausklick zu markieren, kann in AOCOIAC der Pointer auch durch Doppelklick in einen Zeichenstift umgewandelt werden. Im zentralen Fenster lässt sich damit der in der Bildgebung beobachtete Frakturenver-

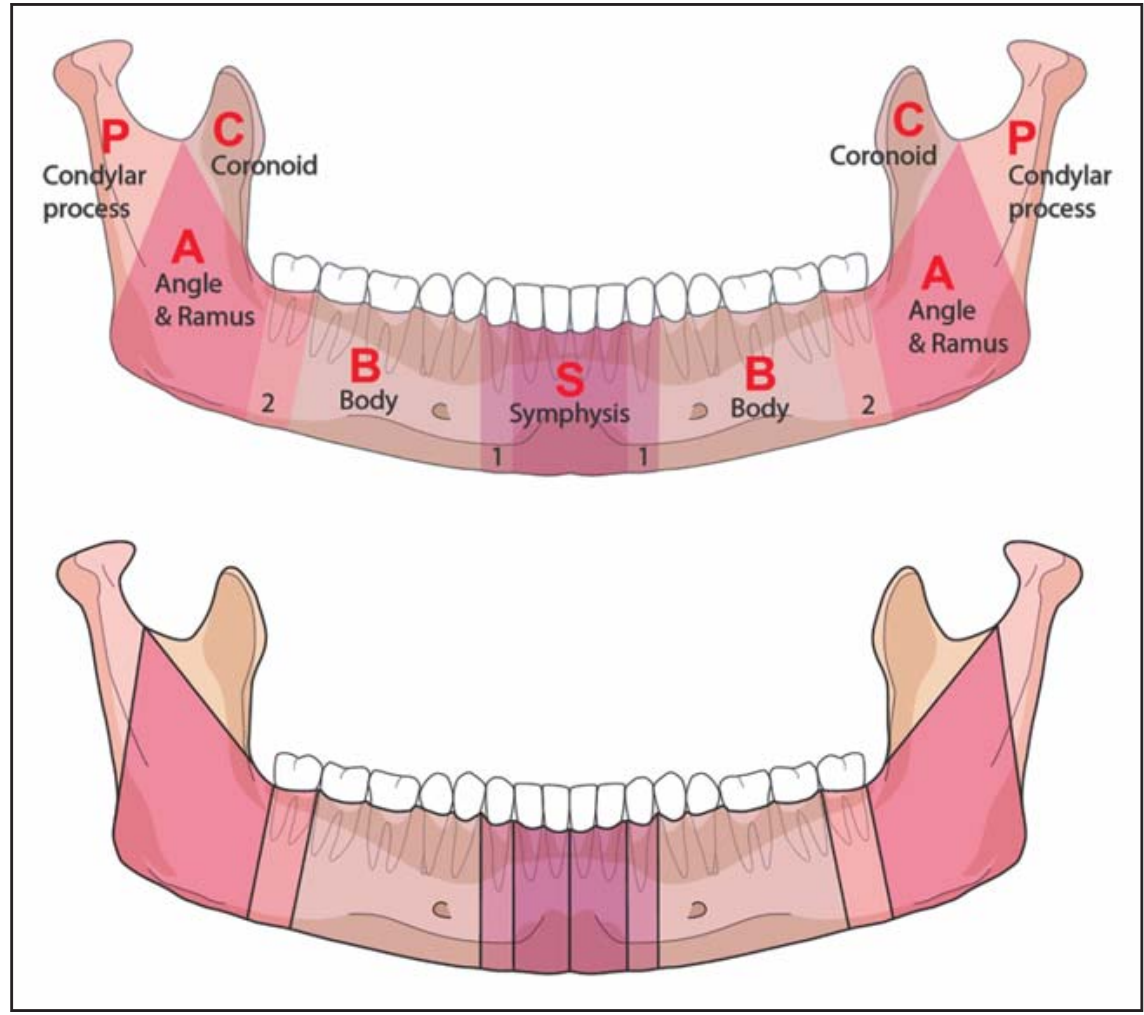

Abb. 7 Mandible Level 2 - Subregions in Panoramaansicht: Unterteilung des Unterkiefer in 9 Untereinheiten. Kennzeichnung mit einem Großbuchstaben zur alphanumerischen Notation: Symphysis (S), Body (B), Angle/Ascending Ramus (A), Coronoid Process (C), Condylar Process (P) (mit freundlicher Genehmigung der AO Foundation, Davos [15]).

lauf einzeichnen. Die Zuordnung einer Fraktur zu den Subregionen nach den o.g. Regeln erfolgt dann automatisiert (Abb. 8b). Mit Ausnahme des Coronoid Process $(C)$ können Frakturen in allen anderen mandibulären Subregionen im Level 3 näher qualifiziert werden.

\section{Mittelgesichtsfrakturen - 92 Midface}

\section{Midface Level 2}

Der Level 2 definiert das Mittelgesicht als den Oberkiefer und mit seinen Flanken verbundene Skelettanteile bis an die Stirnbeinpfeiler (lateral: Sutura zygomatico-frontalis; medial: Sutura fronto-maxillaris bzw. fronto-nasalis). Die Supraorbitalränder und das Orbitadach gehören zum Os frontale und zählen damit zur Schädelbasis.

Das Mittelgesicht einschließlich der internen Orbita (= Orbitawände) wird in Teilbereiche und Subregionen gegliedert und deren Topografie im Einzelnen präzisiert.

In vertikaler Anordnung werden zentrale und laterale Mittelgesichtskompartimente voneinander abgegrenzt (Abb. 9).
Der Oberkiefer oder das zentrale Mittelgesicht besteht aus den 2 Maxillae, welche die Apertura pririformis umgeben und über die frontomaxillären Pfeiler den Kontakt mit der Schädelbasis herstellen.

In Frontalansicht erinnert die Dreiecksform des Oberkiefer bzw. des zentralen Mittelgesichts an die Umrisse einer Pyramide, was bei 3-dimensionaler Betrachtung dann kaum mehr zutrifft.

Das zentrale Mittelgesicht wird in 3 übereinander liegende, horizontale Untereinheiten aufgeteilt (Abkürzung in Großbuchstaben zur Notation in alphanumerischem Code):

- UCM = Upper Central Midface

- ICM = Intermediate Central Midface (= Parapiriform Maxilla/Infraorbital Maxilla)

- $\mathbf{L C M}=$ Lower Central Midface (= Maxillary bodies)

Das obere zentrale Mittelgesicht (UCM) entspricht dem Nasenskelett einschließlich der frontalen Maxillafortsätze. Das intermediäre zentrale Mittelgesicht (ICM) ist mit den parapiriformen bzw. infraorbiatlen Maxillaanteilen gleich- 


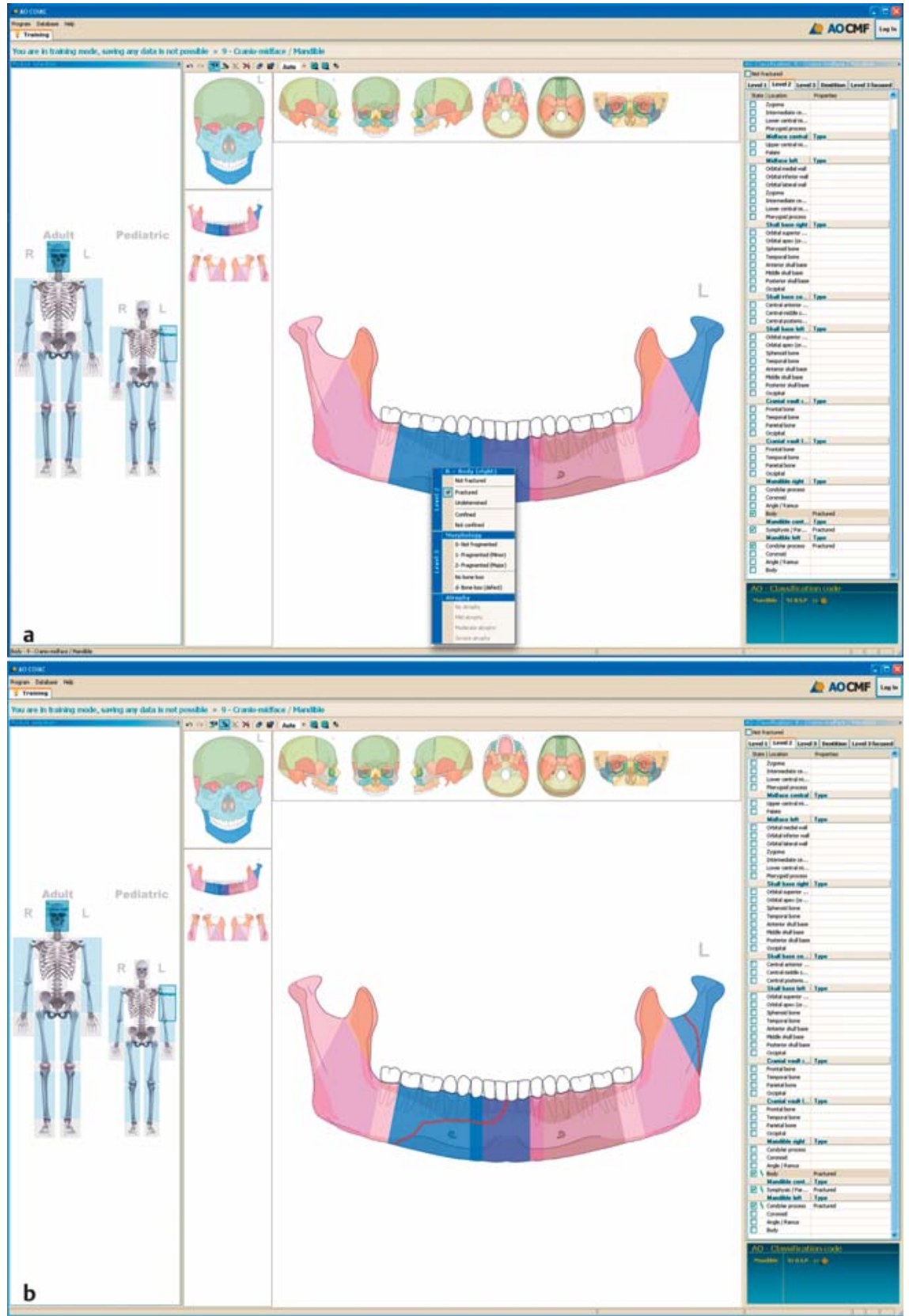

Abb. 8a und b Mandible Icon Level 2 im zentralen unteren Fenster - exemplarisch: bilaterale Fraktur. a Die fragmentierten Subregionen (S, B right, P left) sind blau eingefärbt; die Kennzeichnung kann über direktes Anklicken oder das Pull-down-Menü erfolgen. Nach der Auswahl für Level 2 sind darin die Level-3-Optionen aufgelistet; b eingezeichnete Frakturlinienverläufe; die Fraktur rechts beteiligt A und B und überkreuzt die „Anterior Transitional Zone“.

zusetzen. Das untere zentrale Mittelgesicht (LCM) ist mit den massiveren Knochenstrukturen (Gaumendach, Oberkieferbasis, Alveolarfortsätze $=$ Korpusbereich) des Oberkiefers identisch. Zu beachten ist, dass LCM die Tuberregion bis an die mediale Kante der Fissura orbitalis inferior einschließt.

Das Nasenseptum und Vomer finden erst in Level 3 Berücksichtigung.
Das laterale Mittelgesicht setzt sich aus dem Zygoma/Jochbein und dem Zygomatic Arch/Jochbogen zusammen. Obgleich zwei Drittel des Jochbogens de facto ein Fortsatz des Os temporale/ Schläfenbeins sind, wird er in gesamter Länge in die schematische Einheit $(Z)$ einbezogen und ist in Kombination mit dem Jochbeinkörper zu sehen. Die hintere Begrenzungslinie dieser Einheit verläuft durch den temporalen Ansatz der Kiefergelenkgrube.
Separat von der Zygoma Einheit wird die facies orbitalis/lateral orbital flange des Jochbeins der lateralen Orbitawand zugerechnet und dort lokalisierte Frakturen werden als Frakturen der internen Orbita dokumentiert (Abb. 10)

Der Pterygoid process (Pt)/processus pterygoideus/Flügelfortsatz sowie der Orbital Apex/Apex orbitae/Augenhöhlen Konus/Dorsale Orbita sind zwar Bestandteile des Sphenoids/Os sphenoidale/Keilbeins und gehören somit zur Schädelbasis, werden zur Vereinfachung aber im Level 2 als zwei unabhängige Regionen betrachtet.

Anatomisch stellt der Palate (P)/Hartgaumen keine knöcherne Entität dar, sondern insgesamt tragen 4 Bausteine (Maxilla jederseits mit je einer horizontalen Platte/processus palatinus für die vorderen zwei Drittel und die Lamina horizontalis des Gaumenbeins jederseits für das hintere Drittel) zur Konstruktion des Gaumendachs bei; im Kontext von Level 2 wird dennoch eine einzige übergeordnete Struktur daraus.

Die Interna Orbita, sprich die Orbitawände werden im Level 2 nach ihrer Geometrie ausgewertet, d.h. ohne detaillierten Bezug auf die 7 am Aufbau beteiligten Einzelknochen. Im vorderen Orbitaabschnitt und in der Midorbita $(=$ mittlerer Orbitaabschnitt $)$ sind die 4 Wände in einem viereckigen Querschnitt angeordnet (vgl. Abb.1rc - Jaquiéry et al. - Beitrag in diesem Heft), der sich nach dorsal verjüngt.

Bei 3-dimensionaler Betrachtung entspricht dies einer Pyramiden Konfiguration, deren Basis sich auf den Vordereingang/Aditus ad orbitae projiziert. Das hintere Ende des Orbitabodens geht in die mediale Orbitawand über, sodass die Apex region bzw. der dorsale Orbita-Abschnitt eine Trianguläre Konfiguration bekommt.

Nach diesen Vorgaben setzt sich die interne Orbita folgendermaßen zusammen:

Anterior Orbital Section and Midorbit:

- Orbital superior Wall (Os)/Orbital Roof

- Orbital lateral Wall (OI)

- Orbital medial Wall (Om)

- Orbital inferior Wall (Oi)/Orbital floor

Posterior Orbital Section -

- Orbital Apex (Oa) 


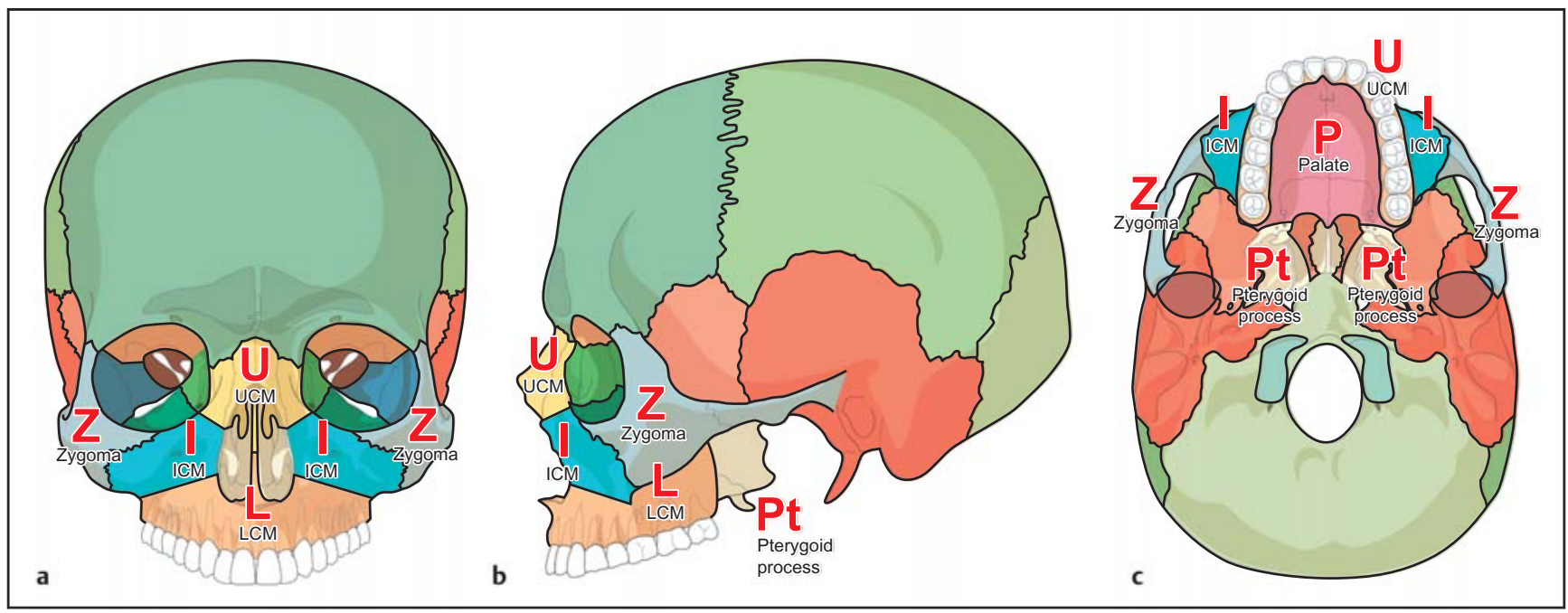

Abb. 9a bis c Midface Level 2 - Subregionen. a Frontalansicht: zentrales Mittelgesicht mit Aufteilung in UCM, ICM und LCM; laterales Mittelgesicht: Zygoma/Jochbein und Jochbogen; b Lateralansicht (links): Pterygoid Process (Pt) processus pterygoideus/Flügelfortsatz - eigentlich zum Keilbein gehörend. Durch farbliche Absetzung als eigenständige Subregion gekennzeichnet; c Kaudalansicht: Palate (P)/palatum durum/harter Gaumen - eigentlich keine Entität, hier aber als In-toto-Struktur konzipiert. Nicht zu verwechseln mit Palatine Bone/os palatinum/Gaumenbein (siehe Text).

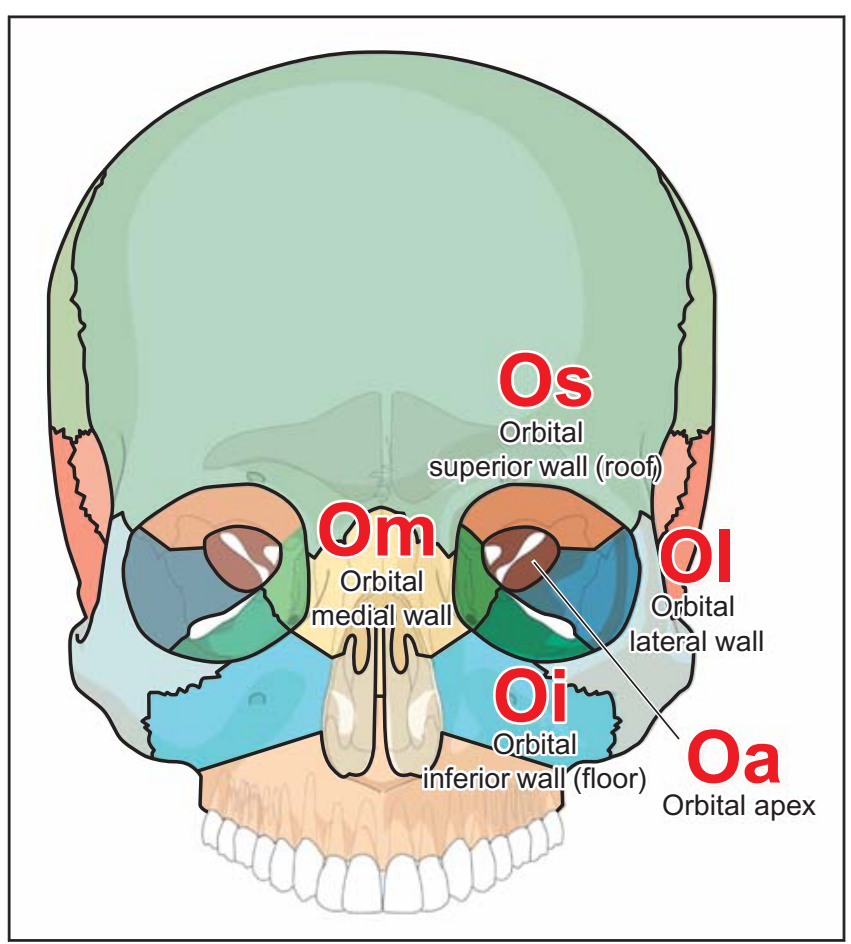

Das Orbitadach (Os) ist, wie bereits erwähnt, ein integraler Teil des Os frontale/Stirnbeins und der Schädelbasis zuzurechnen. Nach dem Level-2-Schema ist es als Untereinheit der Augenhöhle aufzufassen.

Der Apex oder Conus orbitae stellt den posterioren Orbitaabschnitt dar. Dieser beginnt am Hinterende der Fissura orbitalis inferior. Der Apex orbitae wird vom Os sphenoidale gebildet. Der Canalis opticus liegt superomedial zwischen klei-
Abb. 10 Internal Orbit (0) Level 2. Die 5 Subregionen nach geometrischem Konzept (N. s. Text).

nem Keilbeinflügel und Keilbeinkörper. Die Fissura orbitalis superior ist der Spalt zwischen kleinem und großen Keilbeinflügel. Die meisten neurovaskulären Strukturen betreten die Orbita durch diese beiden Einlassöffnungen.

Die Frakturlinien nach der Originalbeschreibung von Le Fort verlaufen entlang der oberen Grenzlinien von LCM (= Le Fort I), UCM + ICM (= Le Fort II) u. UCM + ICM + Zygoma (Le Fort III). Definitionsgemäß (s.o.) handelt es sich um
Blockfrakturen, die immer beide Seiten betreffen und die Processus pterygoidei involvieren.

Zur Anwahl der Original-Le-Fort-Frakturen stehen im Level 2 insgesamt 12 Icons zur Verfügung, in denen die Ausdehnung der 3 Le-Fort-Blöcke jeweils aus 4 Ansichten als farblich unterlegte Felder gezeigt ist (s. Beispiel Abb. 11).

Eine oder mehrere Subregionen im Mittelgesichtsbereich werden selektioniert, um zu dokumentieren, dass innerhalb dieser Zonen oder an deren Grenzen Frakturlinien verlaufen. Bspw. wird eine isolierte Fraktur der fazialen Kieferhöhlenwand durch Markierung von ICM eingegeben.

Die Wahl mehrerer Subregionen steht für Le-Fort-analoge Frakturmuster, d.h. für Frakturkombinationen auf oder in den 3 Le-Fort-Ebenen bzw. Jochbeinfrakturen. So kann das Nebeneinander von Fragmenten im Sinne von Le-Fort-I- und Le-Fort-II-Frakturen jederseits sowie eine einseitige Jochbeinfraktur unilateral gekennzeichnet werden (Abb. 12).

Bei einem Hemi-Le-Fort-I-, -II-, -III-Frakturmuster liegt eine Median-SagittalFraktur vor; die Le-Fort-Ebenen - bzw. repräsentativ dafür LCM, ICM, UCM und Jochbein - sind nur in einer Gesichtshälfte betroffen (Abb. 13). UCM ist im Level 2 immer nur als bilaterales Strukturelement markierbar. 

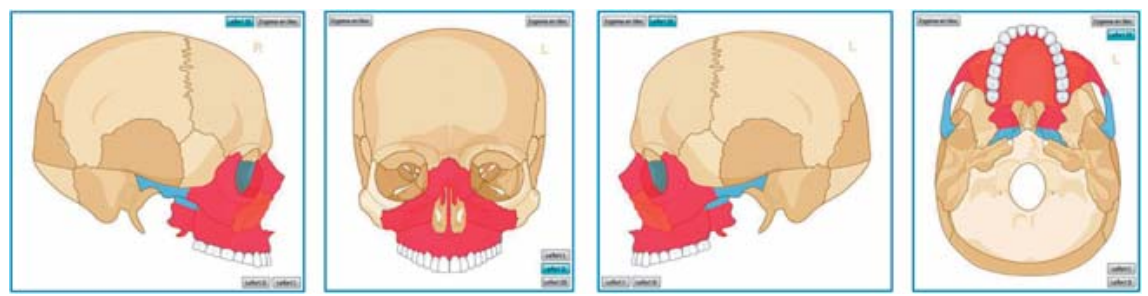

Abb. 11 Le-Fort-III-Fraktur nach der Originalbeschreibung. Integration in 4 Ansichten (lateral rechts, frontal, lateral links, kaudal). Die Selektion in einer Ansicht wird automatisch auf alle zugehörigen Icons übertragen. Die orange eingefärbten ICM weisen darauf hin, dass in diesen Subregionen keine Frakturlinien vorhanden sind.

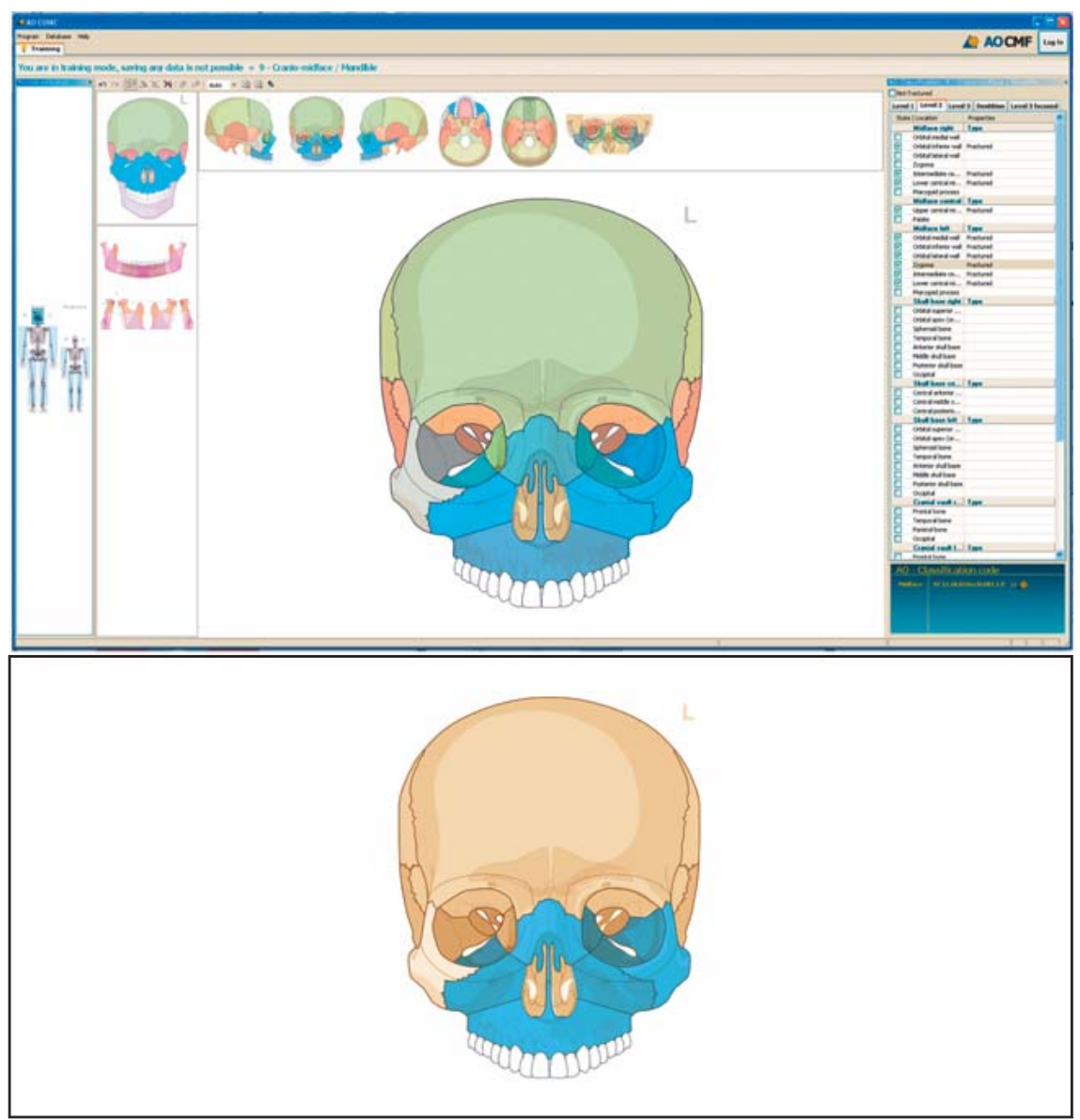

Abb. 12 a und b Midface Frontal View Icon Level 2 im zentralen unteren Fenster - exemplarisch: Le-Fort-analoges Frakturmuster mit Koexistenz von Frakturen innerhalb von LCM, ICM, UCM und Jochbein links oder anders ausgedrückt auf der Le-Fort-I- und -II-Ebene beidseits sowie der Le Fort III einseitig. Die Processus pterygoidei sind nicht involviert. Interne Orbitafrakturen: Orbitaboden rechts, medio-infero-laterale Orbitawände links; a Die Farbcodierung der Subregionen lässt sich in einen Schwarz-Weiß-Modus umschalten, dadurch kommt das Le-Fort-analoge Frakturmuster klarer zum Ausdruck.

Kurz zusammengefasst integriert Midface Level 2 einerseits die Le-Fort-Frakturen und ermöglicht anderseits die Extrapolation auf davon abweichende, aber grundsätzlich analoge Frakturmuster.

Außerdem sind Frakturen der internen Orbita, wenn auch auf eine einfache Architektur reduziert, registrierbar (Abb. 14).

\section{Unterkieferfrakturen - 91 Mandible}

\section{Mandible Level 3}

Mandible Level 3 geht den nächsten Schritt und erweitert die topografische Zuordnung (= Level 2) von Frakturen um die Beschreibung individueller prätraumatischer Befunde (Zahnstatus bzw. Zahnlosigkeit und Atrophiegrade nach
Luhr), die Aufzeichnung von Zahn- und Parodontaltraumen und um die Frakturenmorphologie (hier: Fragmentation). Vorausssetzung dazu ist eine multiplanare und/oder 3-D-Bildgebungstechnik (CT/DVT). In konventioneller Röntgendarstellung und Panoramaschichtaufnahmen ist der Unterkiefer nicht von der Medial- bzw. Dorsalseite beurteilbar - man könnte sagen im „Blacksmith/Farrier View“", da der Anblick ans Hufbeschlagen bei Pferden erinnert. Nur bei Betrachtung beider Ansichten ist der Grad der Fragmentation und das Vorkommen von Sagittalfrakturen im Mandibularbogen sowie in der Angulus/Ramus-Region genau $\mathrm{zu}$ bestimmen (Abb. 15).

Bei partieller oder vollständiger Zahnlosigkeit (Vor-Trauma) lässt sich der Atrophiegrad der Unterkieferalveolarfortsätze bzw. des Unterkiefers mit der LuhrKlassifikation angeben (Luhr et al. 1996) (s. auch Cornelius et al. 2013 - dieses Heft).

Der sichtbare Abbau der UnterkieferVertikalhöhe spielt sich in den Korpusregionen und der Symphyse ab und kann für jede diese Subregionen einzeln angegeben werden. Bei einer Höhe von $>20 \mathrm{~mm}$ liegt definitionsgemäß noch keine Atrophie vor. Die Atrophiegrade darunter folgen der nachstehenden Einteilung:

1 = Mild Atrophy: Vertical Height $>15-$ $20 \mathrm{~mm}$ (Luhr Class I)

2 = Moderate Atrophy: Vertical Height $>10-15$ mm (Luhr Class II)

3 = Severe Atrophy: Vertical Height $\leq 10 \mathrm{~mm}$ (Luhr Class III)

Daraus ergibt sich in den 3 Subregionen ein Grid mit 9 Feldern, das eine Klassifikation aller denkbaren Atrophievarianten zulässt (Abb. 16).

Der vermutliche Vor-Trauma-Zahnstatus im Unter- und Oberkiefer wird nach dem FDI-Schema (Fédération Dentaire Internationale) eingetragen.

Unter Bezug auf diese prätraumatische Ausgangssituation können dann aktuelle Verletzungen der Zahnhartsubstanz (Kronen und Wurzelfrakturen) und des Zahnhalteapparats (Zahnlockerung, Avulsion) für jeden Zahn aufgezeichnet werden (Abb. 17). 


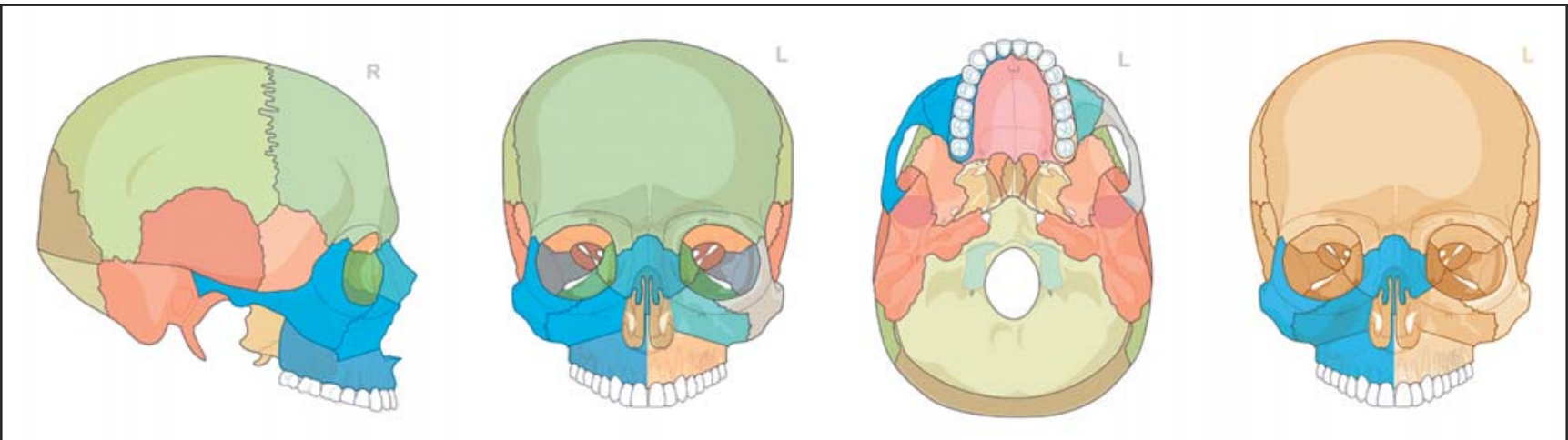

Abb. 13 Hemi-Le-Fort-I, -II-, -III-Frakturmuster rechts. Der Processus pterygoideus ist nicht mitfragmentiert.

Alveolarfortsatzfrakturen sind marginale Knochenaussprengungen, die sich kastenförmig über ein oder mehrere Zahnfächer erstrecken. Sie sind von 2 vertikalen Frakturlinien begrenzt; die verbindende horizontale Frakturlinie verläuft auf Höhe der Wurzelspitzen bzw. an der Grenze zwischen basalem Mandibularbogen und Processus alveolaris. Alveolarfortsatzfrakturen werden dokumentiert, indem der oder die Knochenblöcke in den betroffenen Zahnregionen (nach FDI-Formel) einfach nacheinander markiert werden (Abb. 18).

Zur Angabe des Grades der Fragmentation (Bedeutung siehe Info-Box 1) von Unterkieferfrakturen stehen 3 Kategorien (non-fragmented, minor, major fragmented) zur Verfügung.

Bei der Auswertung der CT-Bilder müssen die äußere und die innere Oberfläche des Unterkiefers überprüft werden (vgl. Abb.15). Der höchste Fragmentationsgrad, ob auf der Außen- oder Innenseite gelegen, ist für die Klassierung bestimmend.

In den mandibulären Subregionen Symphyse und Korpus/Body haben die 3 Fragmentationsgrade folgende Bedeutung (Abb. 19):

- No Fragmentation/Grade 0 bezieht sich auf eine einzelne Frakturlinie ohne oder mit „intermediären Mikrofragmenten“ in ihrem Verlauf (Abb. 19a).

- Minor Fragmentation/Grade 1 steht für „kleine intermediäre Fragmente“, die entweder entlang des basalen Unterkieferrands oder im Bereich des Alveolarfortsatzes verteilt sind. Der Unterkiefer darf dabei nicht in voller Höhe von diesen Fragmenten durchsetzt sein (Abb. 19b)

- Major Fragmentation/Grade 2 bezieht sich auf intermediäre Fragmente kleinst, klein, oder groß -, die sich über die gesamte Vertikalhöhe des

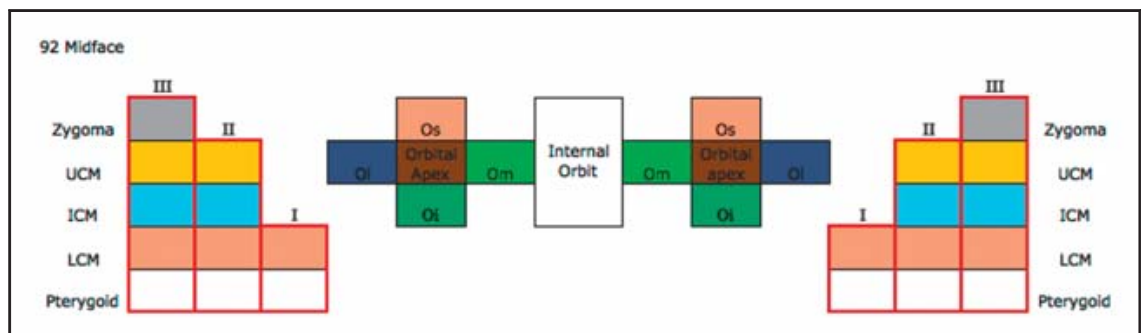

Abb. 14 Subregionen des Mittelgesichts im Treppenschema. Zur Kennzeichnung von Le-FortFrakturen i.e.S. müssen alle Felder bzw. Subregionen in den rot umrandeten Säulen mit den Nummern I, II oder III beider Seiten ausgefüllt sein. Le Fort-analoge-Frakturmuster und deren Terminologie ergeben sich daraus, inwieweit bzw. wie viele Felder/Subregionen innerhalb der LeFort-I, -II-, -III-Säulen beteiligt sind.

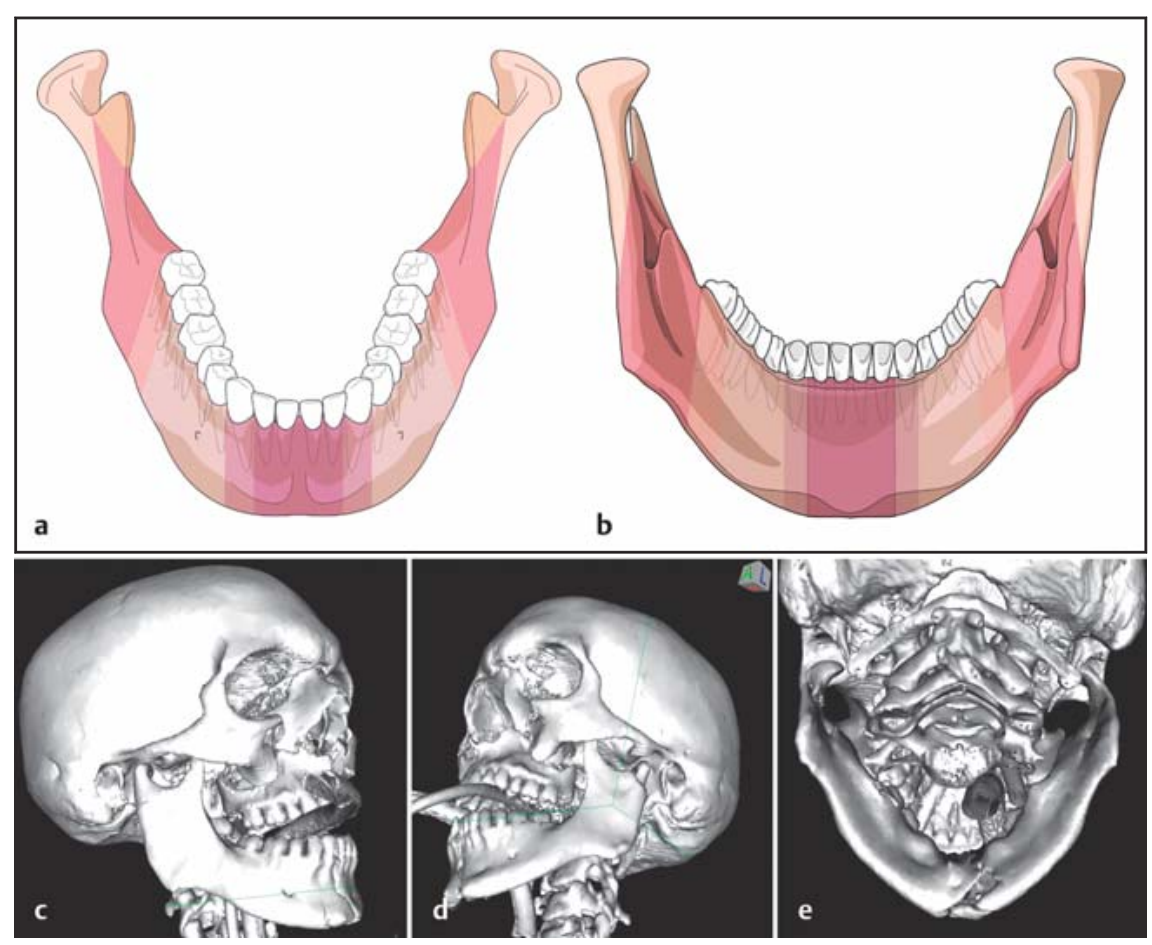

Abb. 15a bis e Mandibula. a Außenansicht; b Innenansicht; $\mathbf{c}$ und d 3-D-CT im Halbprofil von beiden Seiten mit 3-fach-Fraktur des Unterkiefers im Rahmen eines sog. panfazialen Traumas; e „Hufbeschlag“-Ansicht - der basale Keil in der Unterkiefersymphyse beschränkt sich auf die Außenkortikalis. 

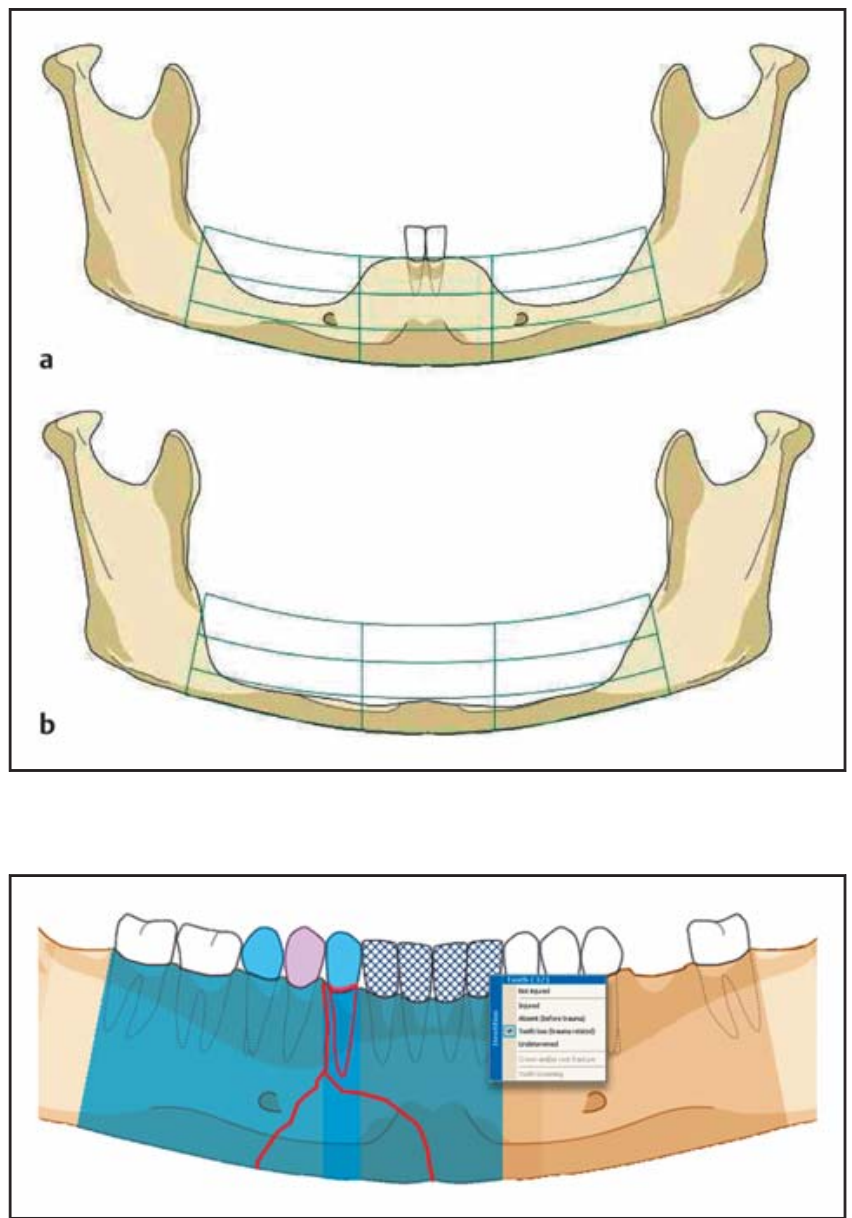

Abb. 17 Dentales und parodontales Trauma in Begleitung einer Unterkieferfraktur (Symphysis/ Body right) mit zugehöriger Farbkennzeichnung. Vor-Trauma-Zahnverluste von 38, 37 und 48, traumatisch bedingte Zahnverluste 32, 31, 41, 42 („cross hatch“), Zahnlockerung 43 („blue crown, red root silhouette“), Kronen/Wurzelfraktur 44 („pink crown“), Verletzung ohne nähere Angabe 45 („,blue crown“). Neben 32 ist das Pull-down-Menü mit der Liste der Auswahlmöglichkeiten gezeigt.

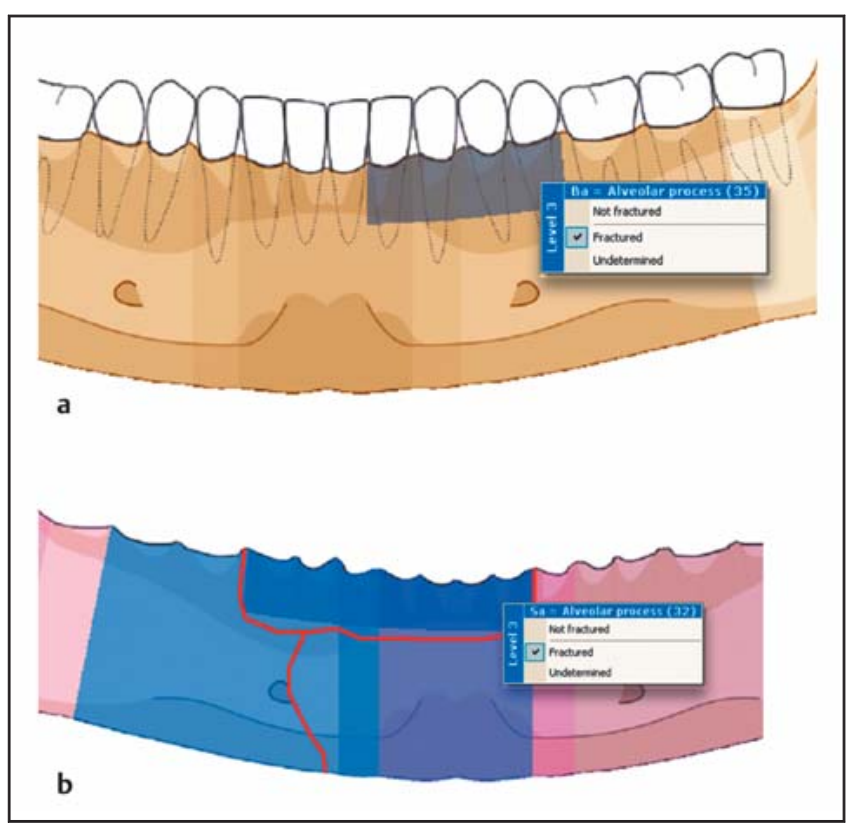

Abb. $16 \mathrm{a}$ und $\mathbf{b}$ eines teilbezahnten und eines zahnlosen Unterkiefers mit Projektion der Luhr-Klassen in Form eines Grids; a Moderate Atrophie im Korpusbereich beidseits, bereich erhalten; b extrem fortgeschrittene Alveolarfortsatzatrophie über dem gesamten Mandibularbogen.
Panoramaansicht Höhe im Symphysen-
Abb. 18 a und $\mathbf{b}$ Alveolarfortsatzfrakturen. a Im bezahnten Unterkiefer Regio 32 bis 35 - Pulldown-Menü neben 35; b im unbezahnten Unterkiefer Regio 45 bis 32; hier wird so verfahren, als seien die Zähne noch vorhanden, außerdem Fraktur (Body right) des basalen Mandibularbogens.

Unterkiefers ausdehnen. In diese Kategorie gehören auch parallel angeordnete Frakturlinien, die ein großes intermediäres Fragment zwischen sich demarkieren (Abb. 19c).

Auf die speziellen Bedingungen zur Beschreibung von Fragmentationsgraden in der Angulus/Ramus-Region wird hier nicht eingegangen (s. AOCOIAC und Cornelius et al. 2013 b).

Ein traumatischer Knochenverlust ( $d=$ defect) im Unterkiefer hat unterschiedliche funktionelle Auswirkungen, je nachdem, wie groß und wo der Substanzverlust lokalisiert ist. Bei einer Defektfraktur wird in der Regel an einen Kontinuitätsverlust im basalen Unterkieferkompartiment gedacht. Für die Klassifikation hier ist jedoch allein ausschlaggebend, ob in der Bildgebung erkennbare ossäre Defizite, ursächlich durch Trauma, vorliegen, ohne weitere Quanti- und Qualifizierung.

Displacement/(deutsch:Dislokation), die 2. wesentliche Variable zur Beschreibung der Frakturenmorphologie, ist bis jetzt nicht Gegenstand der Mandible Level 3Klassifikation, u.a. weil Okklusionsstörungen als Ausdruck eines vielleicht geringfügigen, aber klinisch relevanten Displacements in der Bildgebung nicht feststellbar sein müssen und da sich die Fragmente bei Bewegungen des Unterkiefers leicht weiterverschieben, sodass die Situation in der Bildgebung nicht statisch ist.

\section{Unterkiefer - 91 Mandible}

\section{Kiefergelenkfortsatzfrakturen/} Condylar Process Fractures Level 3

Die Klassifikationsmöglichkeiten für Kiefergelenkfortsatzfrakturen im Mandible Level 3 sind umfassend und vielschichtig (Neff et al. 2013), sodass hier nur die „Essentials“ ausführlich wiedergegeben werden können.

Topografisch werden am Gelenkfortsatz in Bezug auf 3 in Etagen angeordneten Referenzlinien folgende Frakturtypen (vgl. Schiel et al. 2013 in diesem Heft sowie Schiel et al., diese Zeitschrift 2012; S. 194-210) unterschieden (Abb. 20):

- Condylar Head Fractures/Gelenkwalzenfrakturen (Synonyme: Gelenkkopf oder diakapituläre Frakturen)

- Condylar Neck/Gelenkhals - bzw. "Collum"-Frakturen

- Condylar Base Fractures/Gelenkfortsatzbasis-Frakturen 


\section{Fragmentation - Begriffserklärung}

Eine Variable zur Charakterisierung von Frakturen ist die Vielfalt der Bestandteile, Einzelstücke oder Fragmente in der Bruchzone oder die sog. Fragmentation.

Der Begriff Fragmentation wird oft als Synonym für Trümmerung bzw. Comminution verwendet, was seinen Grund darin haben mag, dass letztere Begriffe oftmals als „Fragmentierung der Fragmente“ bzw. „Fragmentation of Fragments" definiert werden.

Im Kontext der AO CMF Fracture Classification wird Fragmentation vielmehr als Oberbegriff verwendet für das Erscheinungsbild bzw. Muster, das durch eine oder mehrere Frakturlinien hervorgerufen wird.

Neben 2 Hauptfragmenten, die durch eine Frakturlinie entstehen, können sog. „intermediate fragments“, also Zwischenstücke vorliegen. Dazu sind 2 oder mehr Frakturlinien notwendig, die untereinander in Verbindung stehen oder sich in direkter Nachbarschaft befinden. Dies gilt gleichermaßen im Unterkiefer, Mittelgesicht und Orbitabereich.

In den mandibulären Subregionen variieren ,intermediate fragments“ in der Größe (Partikel bis massive Zwischenstücke), in der Anzahl und in der Anordnung über die Höhe und Längsausdehnung des Unterkiefers.

Eine Prämolarenkrone dient bei Frakturen im Mandibularbogen als Maßstab, um die Größe von ,intermediate fragments“ unabhängig von verschiedenen Vergrößerungsfaktoren in der Bildgebung 3-dimensional abzuschätzen. Die Schwelle zwischen „minute“ Fragments bzw. kleinsten oder auch Mikrofragmenten, die bei der Festlegung des Fragmentationsgrads keine Rolle spielen, und relevanten „small fragments“ ist dementsprechend durch die Prämolarenkrone als Bezugsgröße gegeben.

Condylar Head (H) Fractures bzw. Gelenkwalzen-Frakturen verlaufen in Sagittalrichtung des Capitulum und werden nach ihrer Beziehung zur „Lateral Condylar Pole Zone“ in 2 Typen unterteilt (Abb. 21):

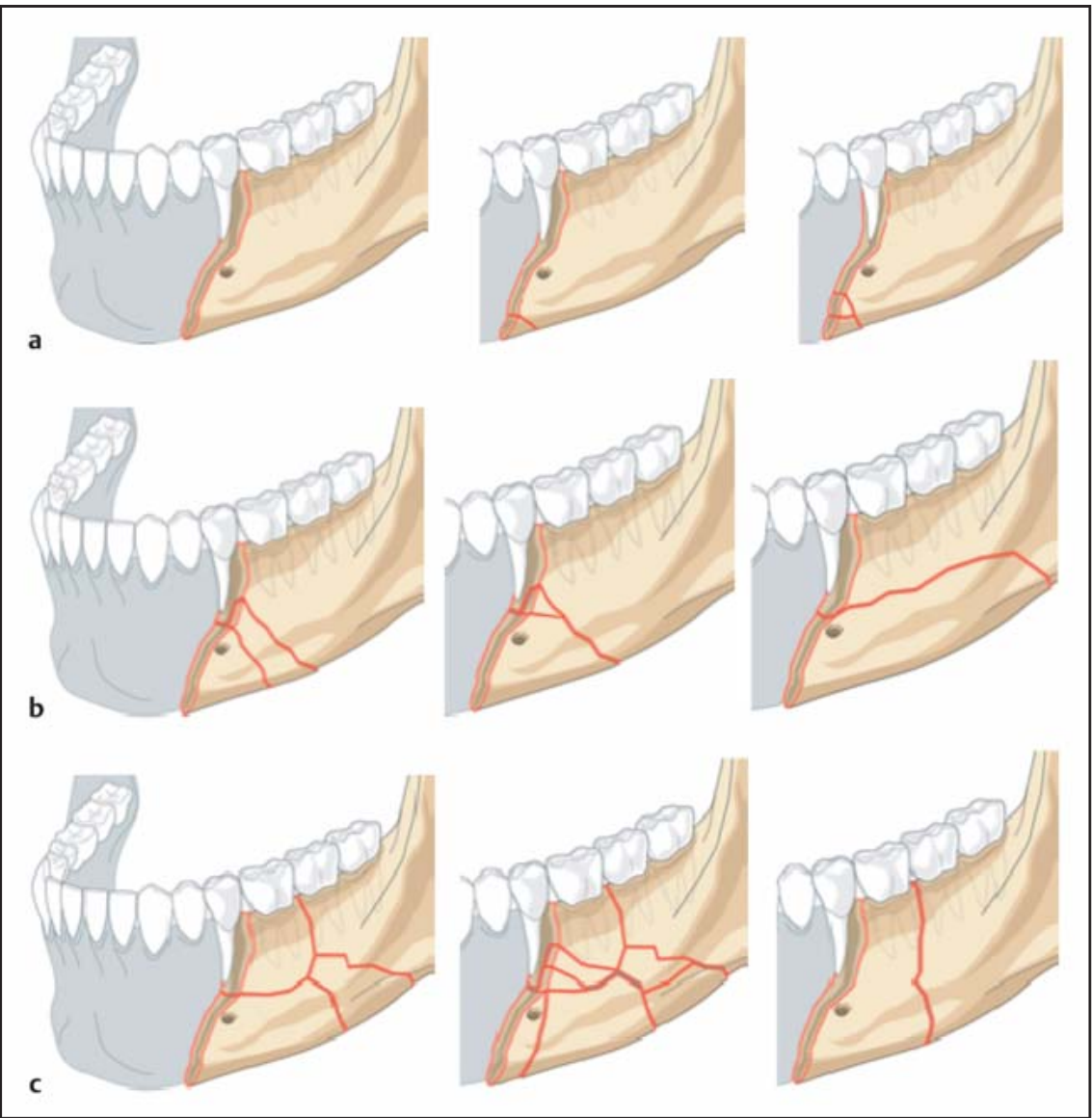

Abb. 19a bis c a No Fragmentation/Grade 0; b Minor Fragmentation/Grade 1; c Major Fragmentation/Grade 2 (Einzelheiten s. Text).

$$
\begin{aligned}
& \text { - } m=\text { Frakturen medial der lateralen } \\
& \text { Polzone } \\
& -p=\text { Frakturen innerhalb oder lateral } \\
& \text { der lateralen Polzone }
\end{aligned}
$$

Der Fragmentationsgrad wird in den bereits bekannten 3 Kategorien angegeben: Die Bewertung als „minor“ oder als „major fragmented“ gründet sich darauf, ob die strukturelle Integrität des Gelenkkopfs durch ein Hauptfragment insgesamt noch erhalten oder aber beeinträchtigt ist (Abb. 21 c, d und f).

Ein Displacement von GelenkwalzenFrakturen spielt sich so ab, dass die Anlagerung des medialen Fragments an das laterale Fragment, d.h. die „Vertical Apposition“, zunehmend verloren geht, weil das mediale Fragment nach anterokaudal disloziert werden kann bzw. einfach abrutscht. Die Vertical Apposition an der Frakturebene wird in 3 Graden abgestuft:

- Grade $0=$ Complete - voll erhaltener Kontakt

- Grade 1 = Partial - nur teilweise aufrecht erhaltener Kontakt
- Grade 2 = Lost - kein Kontakt mehr bestehend

Condylar Neck (N) and Base (B)/Fractures bzw. Collum- und Kiefergelenkfortsatzbasis-Frakturen werden als solche angesprochen, wenn der zugehörige Frakturlinienverlauf zu mehr als 50\% innerhalb der jeweiligen Subregion liegt.

Die Fragmentation in den beiden Subregionen wird in ähnlicher Weise (nonfragmented, minor or major fragmented) beurteilt wie bei Gelenkwalzenfrakturen.

Beim Displacement nach einer Condylar Neck oder Base Fracture sind bei der Klassierung folgende Formen von Fragmentverschiebung (= lat.: dislocatio) zu unterscheiden:

- Sidewards Displacement/Shift to side/ seitlicher Versatz der Fragmentschäfte (= lat.: dislocatio ad latus) (Abb. 22a):

- None $=$ kein Seitversatz, vollständiger Kontakt der Fragmentenden 


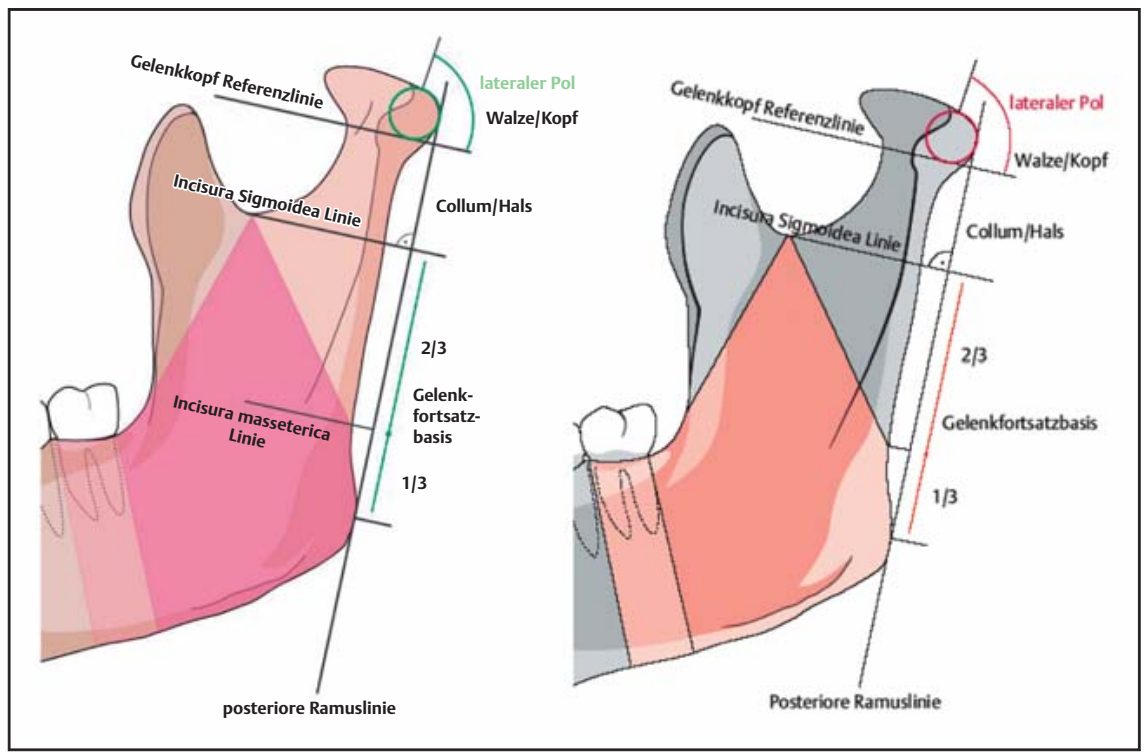

Abb. 20 Lateralansicht des aufsteigenden Unterkieferasts mit Topografie der Subregionen. Die "Incisura-sigmoidea-Linie“ trennt die Gelenkfortsatzbasis/Condylar Base (B) vom Gelenkhals/Collum/Neck (N). Zur Abgrenzung der Gelenkwalze $(\mathbf{H})$ vom Collum $(\mathbf{N})$ wird eine Tangente (Condylar Head Line) kaudal an einen Kreis (grün) gelegt, der den lateralen Pol der Walze umgibt. Das untere Drittel der Gelenkfortsatzbasis kann mit der „Masseteric Notch Line“ von den oberen zwei Dritteln abgegrenzt werden, um damit den hinteren Giebelfuß der Angle/Ramus-Subregion (A) zu definieren. Alle Referenzlinien stehen im Lot auf der posterioren Ramuslinie.

- Partial = seitlicher Versatz mit reduziertem Kontakt der Hauptfragmente in der Frakturebene

- Full/Complete = Versatz um komplette Schaftbreite, kein Kontakt mehr bestehend

- Override/Shortening/Shift in Long Axis with Shortening/Längenverschiebung mit Verkürzung (= lat.: dislocatio ad longitudinem). Voraussetzung ist eine komplette Seitwärtsverschiebung um die volle Schaftbreite von Ramusstumpf und gelenktragendem Fragment. Zum einen kann der Override und seine Richtung (z.B. lateral oder medial über eine Windrose auf Bruchflächenniveau) angegeben werden (Abb. 22 b1, b2 und c), zum anderen können die Auswirkungen auf die gesamte Ramushöhe quantifiziert werden.

- Axial Angulation/Achsenknick (= lat.: dislocatio ad axim): Die Längsachsen der Hauptfragmente (midline axis of ramus and condyle bearing fragment) stehen im einem Winkel zueinander (Abb. 22d). Einteilung der Angulation in 3 Wertebereiche:

$-0=$ None $\left(\leq 5^{\circ}\right)$

- $1=$ Angulation $\leq 45^{\circ}$

$-2=$ Angulation $>45^{\circ}$

Neben der Winkelstellung hat die Angulation auch eine Richtung (Abb. 22e). Da mit jeder größeren Achsenabweichung eine Fehlstellung des Gelenkkopfs in Bezug zur Gelenkgrube einhergeht, ist die Windrose zur Richtungsangabe lateral, medial bzw. anterior, posterior) kranial platziert.

- Distorsion/Rotation/Verdrehung (lat.: dislocatio ad peripheriam). Die Hauptfragmente sind bei dieser Form von Displacement gegensinnig um die Längsachse verdreht. Derartige Rotationsfehlstellungen zwischen den Hauptfragmenten werden im Kiefergelenkfortsatz/Ramusbereich regelmäßig angetroffen (Abb. 22 c). Im Rahmen dieser Klassifikation findet der Begriff Distorsion jedoch Anwendung zur Beschreibung der Kongruenzposition von Kiefergelenkkopf und Gelenkgrube (hier nicht weiter ausgeführt).

$\mathrm{Zu}$ beachten ist, dass die unterschiedlichen Displacement-Formen oftmals kombiniert vorkommen.

Nach einer Condylar Base oder Neck Fracture kann der Gelenkkopf in Normalstellung in der Fossa articularis verblieben sein. Als besondere Form von Displacement kann eine Teil- oder vollständige Luxation ${ }^{2}$ des Gelenkkopfs auftreten, deren Richtung genauso dokumentierbar ist wie bei der Achsenknickung (Abb. 23).

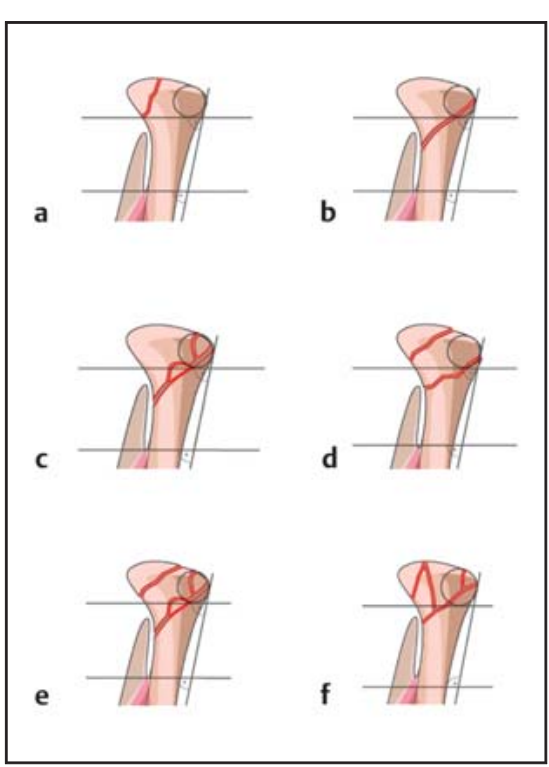

Abb. 21 a bis $\mathbf{f}$ Topografie und Fragmentation von Condylar Head (H)/Walzenfrakturen. a Frakturen medial der lateralen Polzone; b Frakturen innerhalb oder lateral der lateralen Polzone; c Non-fragmented: keine oder ignorierbare Mikro-Intermediärfragmente; d Fragmented minor: eines oder mehrere Intermediärfragmente, strukturelle Integrität durch ein großes Fragment erhalten; e und f Fragmented major: eines oder mehrere Intermediärfragmente, die keine strukturelle Integrität mehr erkennen lassen.

\section{Mittelgesichtsfrakturen - 92 Midface}

\section{Midface Level 3}

Midface Level 3 beschäftigt sich mit den topografischen Subregionen des Mittelgesichts noch intensiver als Level 2.

Im zentralen Kompartiment des Mittelgesichts wird bspw. das UCM, bestehend aus Nasenskelett und den nasofrontalen Maxillafortsätzen bzw. medialen Orbitarändern, in seine anatomischen Komponenten gegliedert, die einzeln anwählbar sind (Abb. 24). Oder die Nasenscheidewand wird in loser Übereinstimmung mit der Verbindungslinie der Lamina perpendicularis des Siebbeins und

\footnotetext{
2 Der Begriff „Luxation“ ist im angolamerikanischen Sprachraum nicht gebräuchlich. Vielmehr ist „Dislocation“ die Wortgebung, mit der ausgedrückt wird, dass der Gelenkkopf außerhalb der Gelenkgrube befindlich ist. Für die lateinisch/deutsche Bezeichnung „Dislokatio(n)“ = Verschiebung/Verlagerung steht in Englisch „Displacement“. Verwirrspielen sind Tür und Tor geöffnet. In der AO-CMF-Klassifikation bezieht sich „Dislocation“ exklusiv auf die vollständige Luxation des Gelenkkopfes aus der Fossa articularis.
} 


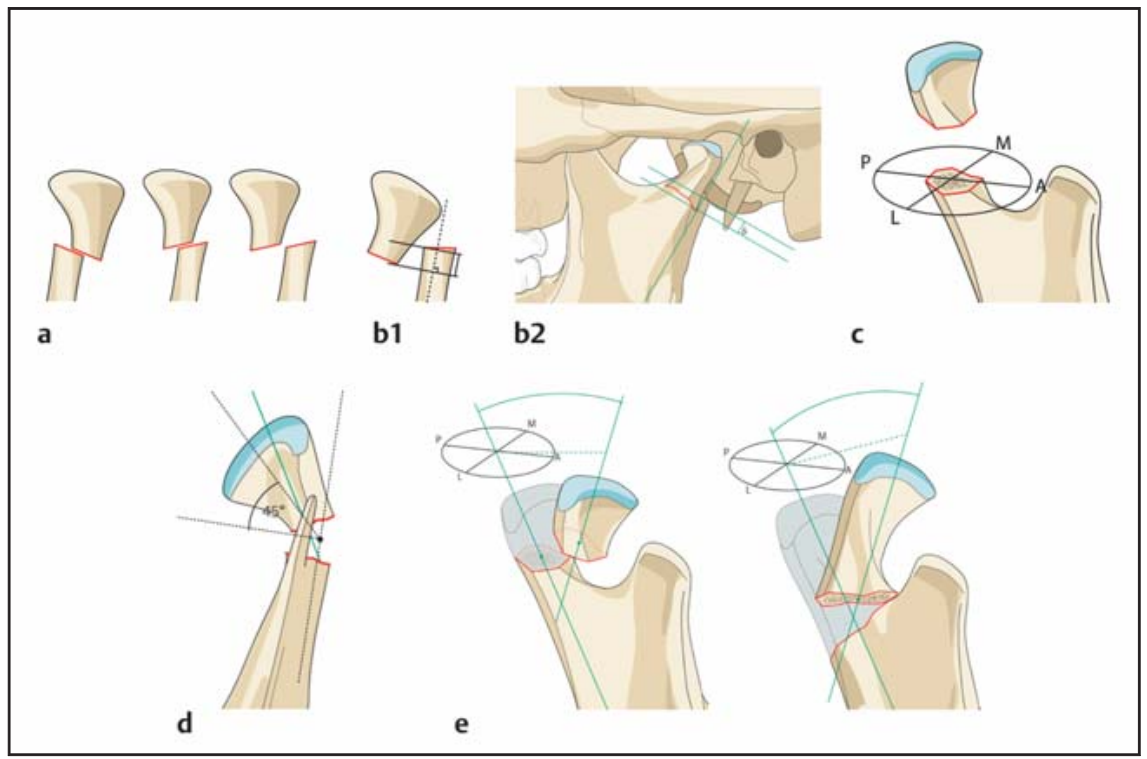

Abb. 22a bis e a Sidewards Displacement des Condylar Process: Partial und Complete in Dorsalansicht; b1 Medial Override in Dorsalansicht; b2 Lateral Override in lateraler Ansicht - der Gelenkkopf sitzt nicht mehr vollständig in der Fossa articularis; c Windrose auf Höhe der Bruchfläche zur Festlegung des Sideward Displacements nach anterior, posterior bzw. lateral, medial und daraus resultierenden Zwischensektoren; d Axial Angulation mit Skizzierung der Winkelbereiche; e Windrose auf Höhe der Gelenkwalze zur Festlegung der Richtung einer Axial Angulation.

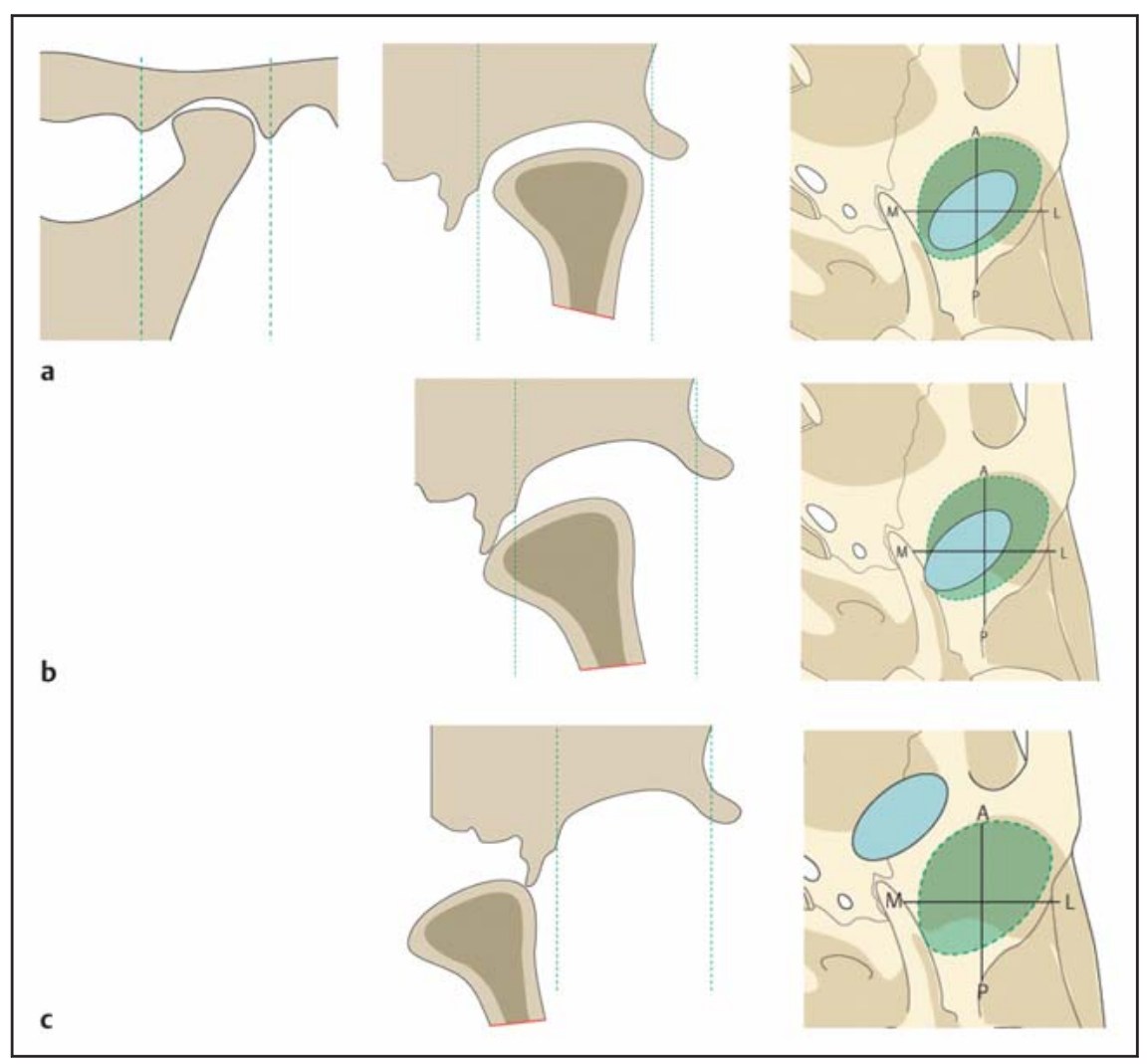

Abb. 23a bis c Displacement des Gelenkkopfs in Relation zur Fossa articularis (Sagittalebene, Frontalebene und Aufsicht von basal auf die Gelenkgrube, Fossagrenzen: grün; Umriss Kopf: blau, Fadenkreuz zur Richtungsangabe). a „No displacement“ (Grade 0); b „Displacement“ (Grade 1): Gelenkkopf befindet sich teilweise außerhalb der Fossagrenzen - hier im postero-medialen Sektor; c „Dislocation“ (Grade 2) - vollständige Luxation² des Gelenkkopfs aus der Fossa - hier nach anteromedial.
Vomer in ein Upper und Lower Nasal Septum vertikal halbiert.

Auf Basis des verfeinerten Layouts der Subregionen können typische Frakturentitäten wie Nasenskelett- und NasoOrbito-Ethmoidalfrakturen (NOE) oder auch Frakturen des Jochbeinkomplexes in ihren Varianten sehr akkurat dokumentiert werden.

Das Jochbein (Zygoma)/Jochbogen-Ensemble entspricht dem lateralen Mittelgesichtskompartiment. Während Zygoma und Zygomatic Arch im Midface Level 2 zu einem Baustein zusammengefasst waren, bringt Level 3 eine minutiöse Aufteilung dieser Region unter Berücksichtigung der 5 Fortsätze, die das Jochbein mit der Stirn, dem großen Keilbeinflügel, der Maxilla im Bereich des Infraorbitalrands sowie der Crista zygomatico maxillaris und dem Schläfenbein verbinden (Abb. 25).

Die Grenzen dieser Subregionen werden im Tutorial Midface Level 3 (Cornelius et al. 2014c) anatomisch genau definiert.

Um den Verlauf und die Anzahl der Frakturlinien exakter anzugeben, werden auch der Palate/Hartgaumen und die Processus pterygoidei (vertikaler vs. horizontaler Verlauf) im Level 3 weiter aufgeteilt.

Gleichartig wie im Unterkiefer lässt sich der Atrophiegrad der Oberkieferalveolarfortsätze bei partieller oder vollständiger Zahnlosigkeit (Vor-Trauma) in 3 Klassen beschreiben (Abb. 24):

1 = No or mild atrophy: Vertical height $\geq 11 \mathrm{~mm}$ 2 = Moderate atrophy: Vertical height $6-10 \mathrm{~mm}$ 3 = Severe atrophy: Vertical height $\leq 5 \mathrm{~mm}$

Zur Dokumentation des Vor-TraumaZahnstatus wird, wie im Unterkiefer, das FDI-Schema (Fédération Dentaire Internationale) verwendet.

Aktuelle Verletzungen der Zahnhartsubstanz und des Zahnhalteapparats lassen sich dann mit Beachtung der prätraumatischen Ausgangssituation mit den gleichen Markierungen wie im Unterkiefer (Abb. 17) festhalten.

Alveolarfortsatzfrakturen im Oberkiefer werden nicht anders als im Unterkiefer aufgezeichnet. Wenn die obere bzw. horizontale Frakturlinie dabei in die vordere Kieferhöhlenwand, den Hartgau- 


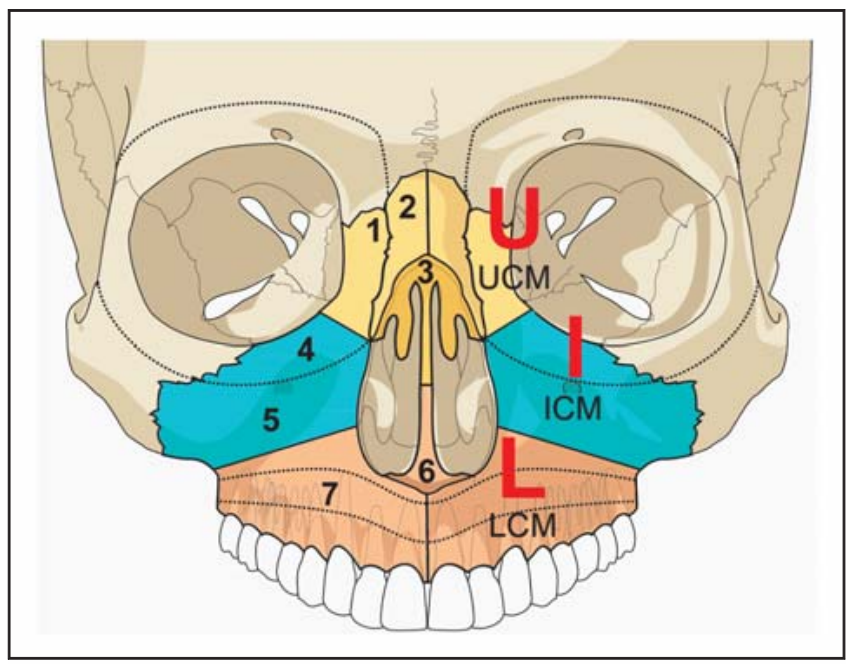

Abb. 24 Zentrales Mittelgesicht und Unterteilung von UCM, ICM und LCM in weitere Subregionen - die Orbitaränder/Orbital Rims sind als kontinuierlicher äußerer Ring um die Öffnung zur Augenhöhle eingezeichnet. 1 Frontonasal maxillary Process/medial orbital Rim (Rm); 2 Nasal Bone/Os nasale; $\mathbf{3}$ Upper nasal Septum; 4 Medial Part of inferior orbital Rim (Ri)/medialer Teil des Infraorbitalrands; 5 Facial antral Wall/faziale Kieferhöhlenwand; 6 Lower nasal Septum; 7 Oberkiefer-Alveolarforsätze mit Kennlinien der Atrophiegrade bei Zahnlosigkeit.

men und/oder den Nasenboden eintritt, wird neben dem alveolären Knochenblock auch das LCM, ICM oder der Hartgaumen als mitbetroffen angeklickt.

Ein Hauptmerkmal von Midface Level 3 ist die Frakturenmorphologie und ihre Eigenschaften (Fragmentation, Displacement und Bone Loss).

Die Morphologieeigenschaften können in sämtlichen Subregionen der Mittelgesichtskompartimente aufgezeichnet werden. Fragmentation wird wechselweise durch die Vorgabe „non-fragmented“ vs. „fragmented“ notiert.

Bei einer „non-fragmented“ Fracture handelt es sich um die Fraktur einer Subregion mit singulärer Bruchlinie, die entweder linear/gerade oder kurvig/gebogen verlaufen kann. Bei einer „fragmented" Fracture liegen multiple Bruchlinien $(\geq 2)$ in einer Subregion vor.

Displacement hat eine einfache Definition: die Fragmente haben die originäre Ausrichtung verloren und befinden sich nicht mehr in der ursprünglichen Fluchtung (= lack of alignment).

Die Vorgaben für Displacement sind wieder alternierend - "non-displaced“ vs. "displaced“.

Knochendefizite lassen sich ebenfalls mit einer dualen Wahlmöglichkeit „no bone loss“ vs. „bone loss“ aufzeichnen.
Die Suturen ZSS und ZFS werden mit den Attributen „non-fractured“, „fractured“ oder „undetermined“ versehen. In der Mehrzahl der Fälle halten sich die Frakturenlinenverläufe linear an diese Suturen. Falls eine Fragmentierung auftritt, werden die benachbarten Subregionen als „fragmented“ eingestuft.

Das Ausgangsszenario für die endlose Zahl von Traumavarianten in den Bereichen von NOE und Jochbein/Zygoma/ Jochbogen-Ensemble sind En-bloc- bzw. Integral-Single-Piece-Frakturen, wobei die Bruchlinien den anatomischen Suturen folgen. Diese En-bloc-Frakturen können über zugehörige Icons registriert werden. Für alle komplexeren Fraktursituationen ist ein Katalog mit Frakturmustern zum Vergleich und zur Wiedererkennung sowie mit Algorithmen zur Auswahl der passenden Morphologieeigenschaften in den Tutorials als auch im Hilfe-Menü der AOCOIAC Software Application hinterlegt.

\section{Mittelgesicht - 92 Midface/ Orbita Level 3}

\section{Orbita - Frakturen des Rahmens und der Wände}

\section{Orbita/Orbitawände}

Auf der Basis der geometrischen Betrachtungsweise und Einteilung der Orbita in wenige Subregionen - Orbitaorbital Rims (R)/Orbitaränder, Internal Orbit (4

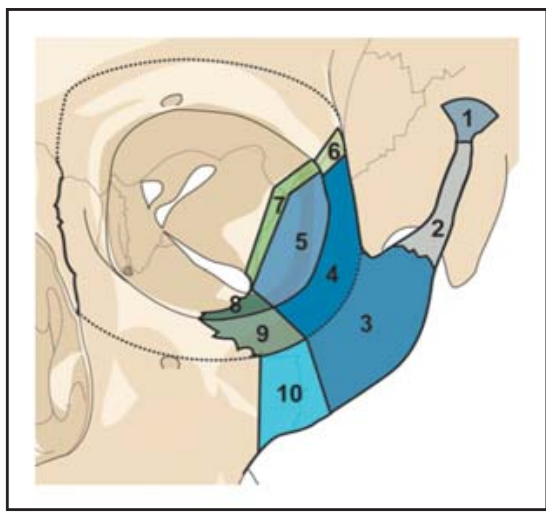

Abb. 25 Laterales Mittelgesicht (Zygoma) und Unterteilung in weitere Subregionen: 1 Temporal Origin of zygomatic Arch; 2 Zygomatic Arch/Jochbogen; 3 Zygoma Body/ Jochbeinkörper; 4 Zygoma Body - Lateral orbital $\operatorname{Rim}(\mathbf{R I}) ; \mathbf{5}$ Lateral orbital Wall - anterior Part/Facies orbitalis ossis zygomatici; 6 Zygomatico-frontal Suture (ZFS); 7 Zygomaticosphenoidal Suture (ZSS); 8 Zygoma Part of antero inferior orbital Wall; 9 Zygoma Part of inferior orbital Rim (Ri); 10 Zygomatico maxillary Crest Area (ZMC).

Walls)/Orbitawände, orbital Apex (A)/Orbitaspitze - in Midface Level 2 wird hier zusätzlich eine Dreiteilung in anteriorposteriorer Richtung vorgenommen.

Da dazu eindeutige, im CT identifizierbare Landmarken notwendig sind, wurden der vorderste Punkt der Fissura orbitalis inferior (= anterior loop of the inferior orbital fissure) und der zum Keilbein gehörende Knochensteg über dem Foramen rotundum als Bezugspunkte gewählt (Abb. 26). Dieser Steg bildet eine Brücke zwischen Keilbeinkörper und großem Keilbeinflügel, zugleich trennt er die Fissura orbitalis inferior von der Fissura orbitalis superior (= bony confluence between inferior and superior orbital fissures). Eine eigene lateinische Bezeichnung in der anatomischen Nomenklatur für diesen Steg existiert nicht, in englischer Terminologie wird er gelegentlich als „maxillary strut" beschrieben.

Im Klinikjargon ist vielfach von einem vorderen, mittleren und hinteren Drittel sowohl der Orbita als auch des kürzeren Orbitabodens die Rede. Die im OrbitaLevel 3 definierte Dreiteilung in eine „anterior orbit“, „midorbit“ und „posterior orbit“ resp. Orbital Apex folgt jedoch keiner proportionalen Metrik und die Segmente haben sicherlich eine unterschiedliche Tiefe in der Sagittalachse.

Orbitafrakturen können die Einzelknochen im Bereich der Orbitaränder und 
Orbitawände in ganz unterschiedlichen Mustern involvieren und im Extremfall eines High Energy Impacts alle 4 Wände und sämtliche Ränder in Mitleidenschft ziehen.

Unter klinischen Gesichtspunkten werden Kombinationen aus Rand- und Wandfrakturen in folgenden Entitäten bzw. Zonen zusammengefasst:

- Supraorbitalränder und Orbitadach

- NOE-Frakturen im oberen Mittelgesicht (UCM)

- zygomatico-orbitale Frakturen (Jochbein und inferolateraler Orbitarand = Hauptkomponenten)

Bei „Blow out“- oder „Blow in“-Frakturen sind isoliert nur die Orbitawände (Orbitaboden $>$ mediale Orbitwand $>$ Orbitadach) und nicht der Rahmen betroffen (= fractures of the internal orbit).

Abweichend von den zuvor genannten klinischen Zonen werden die Orbitaränder/Orbital Rims ( $R$ ) in 4 geometrische Segmente aufgeteilt: medial (Rm), inferior ( $\mathrm{Ri})$, lateral (Rl), superior (Rs). Anatomisch tragen 4 Einzelknochen (Tränenbein/Os lacrimale, Maxilla, Jochbein, Stirnbein/Os frontale) zu diesen RimSegmenten bei.

Deshalb wird das Ri-Segment bzw. der Infrorbitalrand nochmals in einen maxillären und einen Jochbeinanteil (maxillary and zygoma part) und das Rm-Segment in ein maxilläres und ein frontales Teilstück gegliedert.

Nach Partitionierung der internen Orbita in ein Gefüge aus insgesamt 12 Subregionen im anterioren Teil, Midorbita und Apex ergibt sich pro Orbita einschließlich der beiden Suturlinien ZFS und ZSS und der 6 Randabschnitte ein umfangreiches Mosaik aus 20 Bausteinen, in dem sich jedes denkbare Frakturmuster abbilden lässt (Abb. 27).

Die morphologische Eigenschaft Fragmentation erhält in der internen Orbita besondere Bedeutung, weil die Knochenwände von pneumatisierten Hohlräumen (Nasen-Nebenhöhlen-System) umgeben sind, in die Fragmente disloziert (Blow out) werden können, sodass Defektsituationen entstehen. Insofern kann die Fragmentation folgende Attribute aufweisen:

- Linear fractures/lineare Einzelfrakturen, die ohne oder mit einer Dislokation („non-displaced“ vs. „displaced“) einhergehen können. Zusätzlich dür-

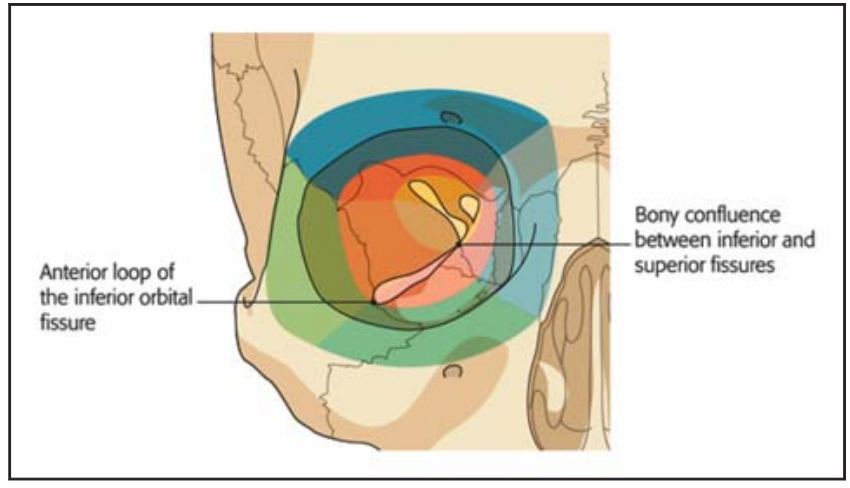

Abb. 26 Landmarken zur Dreiteilung der Orbita-Kavität (anterior, Midorbita, Post/Apex) (s. Text).

fen kleine Fragmente vorhanden sein, die jedoch den linearen Frakturverlauf nicht grundlegend ändern.

- Defect-like lamellar fractures/lamelläre Frakturen, wobei die Fragmente immer disloziert/displaced sind.

- Defects/Defekte, wobei die Fragmente immer disloziert/displaced sind, und zwar soweit, dass der Weichgewebeinhalt der Orbita keine ausreichende Abstüzung mehr hat und in das $\mathrm{Na}$ sen-Nebenhöhlen-System prolabiert. Die dislozierten Knochen zeigen dabei keinen oder nur noch punktuelle Kontakte (offene Falltür, Scharnier) mit unverletzten Knochenpartien

Unter Displacement bei Frakturen einer Orbitawand ist jede strukturelle Abweichung von der typischen Formgebung und Oberflächenkonturierung (vgl. dazu Jaquièry et al. 2013 - dieses Heft) zu verstehen.

Defect-like lamellar Fractures und Defect Fractures mit Displacement des sog. „posteromedial bulge“ oder auch „key area" genannt sind von Interesse, da diese Knochenkonvexität entscheidend für die Bulbus-Position verantwortlich ist. Die Key Area ist in der Midorbita im Übergang zwischen Orbitaboden und medialer Orbitawand lokalisiert.

Der Midface/Orbita Level 3 erlaubt, die Beteiligung/Involvement wichtiger intraorbitaler Strukturen ergänzend $\mathrm{zu}$ dokumentieren. Unteren anderem zählen dazu:

- Inferior OrbitaI Fissure (IOF)/Fissura orbitalis inferior: setzt sich in den Retromaxillarraum und die Fossa infratemporalis fort. Orbitabodenfrakturen können sich bis in den Medialrand der Fissur ausdehnen und führen dann zu einer Vergrößerung der Durchlassöffnung mit konsekutiver Zunahme des Orbitavolumens (Enophthalmus).
- Posterior Ledge $(\mathrm{PL}) \approx$ Facies orbitalis des Gaumenbeins (!) am Dorsalende des Orbitabodens. Die PL ist bei defektartigen oder Defektfrakturen mit wenigen Ausnahmen erhalten und dient bei der periorbitalen Dissektion als Landmarke sowie als Auflage (Ledge = Sims, Ablage) für Rekonstruktionsmaterial (z. B. Titan Meshes).

- Inferior orbital buttress (IOB)/Infero medial orbital Strut/Transition(s) Zone: Knochenverdickung um die Sutura ethmoido-maxillaris, die als Verstrebungspfeiler zu werten ist. Diese entspricht der Trennlinie bzw. dem Übergang zwischen Orbitaboden und medialer Orbitawand, daher der Name Transitionszone. Die Grundlamelle der Bulla ethmoidalis bedingt die Knochenverdickung. Falls die IOB bei 2Wand-Frakturen von Orbitaboden und medialer Orbitawand erhalten ist, erleichtert das die Reparatur bzw. Rekonstruktion erheblich.

- Optic Canal/Canalis opticus: Kanal für Sehnerven und A. ophthalmica dorsosupero-medial im Apex orbitae, durch den Optic Strut von der Fissura orbitalis superior getrennt.

- Greater Wing of Sphenoid (GWS)/großer Keilbeinflügel: Baustein der lateralen Orbitawand in Midorbita und posteriorem Segment, wird nachposterior zunehmend dicker bei Frakturen meist intrakranielle Beteiligung (mittlere Schädelgrube).

- Medial canthal Ligament Insertion/ Ansatz des inneren Lidbands: im Bereich um den Ductus lacrimalis und der Crista posterior des Tränenbeins lokalisiert. Ein Ausriss des Lidbands kann nur klinisch oder intraoperativ festgestellt werden. In der CT-Bildgebung ist eine Multifragmentierung im Rahmen von NOE-Frakturen jedoch hinweisend.

Die unendliche Anzahl möglicher Frakturvarianten um und in der Orbita macht 


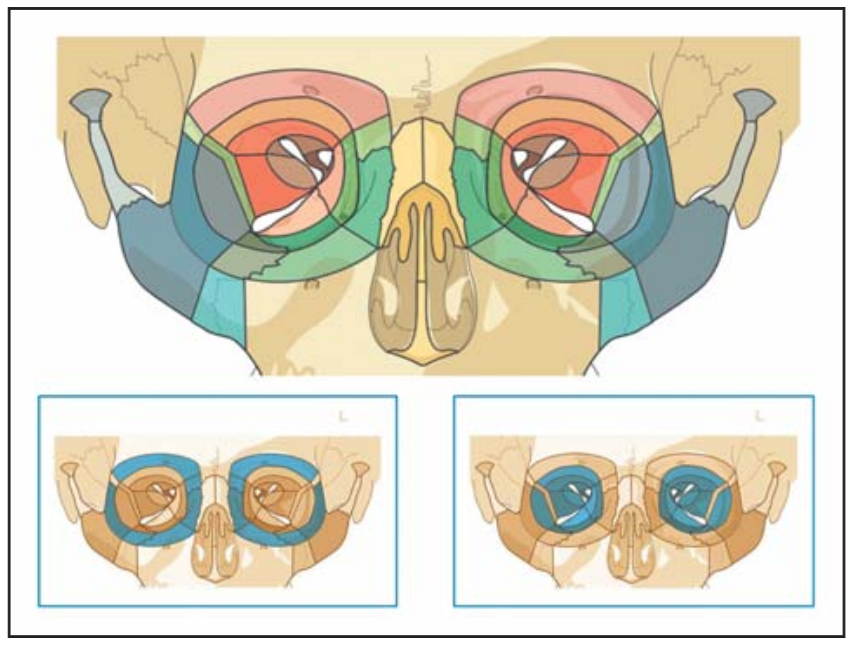

Abb. 27 Strukturelle Aufteilung von Orbitarändern und Orbitawänden bzw. Apex in 20 Bausteinen pro Orbita. Die faziale Kieferhöhlenwand (s. Abb. 24) und die Zygoma-Subregionen (s. Abb. 26) sind in das Dokumentationsschema integriert. ZFS und ZSS sind strenggenommen keine Untereinheiten, sondern Bruchkanten mit i. d. R. linearem Frakturlinienverlauf. Zur Verdeutlichung der Zugehörigkeit der Subregionen sind der Ring aus Orbitarändern und die Orbitawände untenstehend in blauer Farbmarkierung wiedergegeben.

wie im Zygoma/Zygomatic Arch-Ensemble die Erstellung eines Katalogs mit den wichtigsten Frakturmustern erforderlich, um gewisse Normen dafür vorzugeben, wie diese in der CMF-Trauma-Software-Applikation durch passende Angabe der Subregionen von Fragmentation und Displacement zu kennzeichnen sind. Startszenarien für alle weiteren Permutationen werden dabei isolierte Orbitawandfrakturen (1-Wand-Frakturen des Orbitabodens oder der medialen Wand) und aufgrund der Häufigkeit ihres Vorkommens Jochbeinfrakturen mit Beteiligung der Orbitawände sein, deren Komplexität mit einer Zunahme an Frakturlinien und Ausdehnung in benachbarte Subregionen steigt (Abb. 28).

\section{Fazit und Zukunftsperspektiven}

Die 3. Generation AO CMF Fracture Classification zusammen mit der AOCOIACSoftware-Applikation bietet eine umfassende Dokumentationsplattform für knöcherne Verletzungen im Unterkiefer, Kiefergelenkfortsätzen und Mittelgesicht einschließlich der Orbitawände (Schädelbasis und Schädeldach sind hier nicht ausgeführt). Die jetzt vorliegende Klassifikation hat einen langen Weg verschiedener Entwicklungsphasen, Versuchsstadien und Irrtümern hinter sich und stellt sicherlich immer noch keine perfekte Lösung dar.

Dennoch bedeutet die Klassifikation in der augenblicklichen Version einen wichtigen Schritt vorwärts, sie stützt sich auf Bildgebungsdaten, bedient sich zur Dokumentation und Kommunikation moderner interaktiver Computer- und Informationstechnologie und umfasst erstmals den gesamten CMF-Bereich.

Dabei wird ein pragmatischer Ansatz verfolgt: für die topografisch anatomischen Module sind 3 Präzisions-Level definiert, die unterschiedlichen Ansprüchen genügen.

Das Midface-Level-2-Modell integriert die Original-Le-Fort-Klassifikation und kann dazu analoge Frakturenszenarios darstellen, indem die involvierten Subregionen markiert werden. Wie die Berücksichtigung der Le-Fort-Frakturtypen zeigt, ist die neue AO CMF Fracture Classification keine neue Erfindung, sondern gründet auf bekannten und gut etablierten Vorläufern, die sich lohnen, wiederentdeckt zu werden. Das kommt grundsätzlicher Kritik und Kontroversen um das Design und die Leistungsfähigkeit der neuen Version selbstverständlich nicht zuvor. So sind die Grenzen der Subregionen durchaus diskutabel (z.B. Angle/Ramus Region) oder Wortungetüme wie "fragmentierte Fraktur" sprachlich anfechtbar. Ferner ist die AOCOIACCMF-Software-Applikation mitnichten so intuitiv und selbsterklärend wie gewünscht. Und die Menge an Informationen in Level 3 stößt an die Grenzen der Darstellbarkeit durch Icons und Farbgebung auf dem Bildschirm, sodass eigentlich aufwendige und kostenspielige Animationstechniken mit Objekttrans-

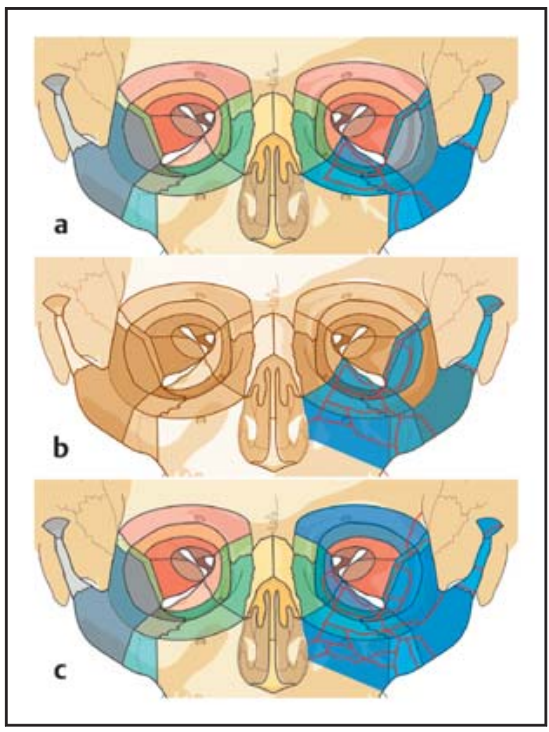

Abb. 28a bis c Jochbeinfraktur links mit Orbitafraktur(en) von zunehmender Komplexität - AOCOIAC-Simulation im Sinne eines Crescendo von Fragmentation und topografischer Ausdehnung über benachbarte Subregionen. a Vergleichsweise „einfache“ Jochbeinfraktur mit Stückbruch des Infraorbitalrands und der ZMC; b Frakturmuster a + Abscherfraktur im Jochbogenursprung, lamelläre Fraktur der Zygomatic orbital Flange, Mehrfachfraktur der fazialen Kieferhöhlenwand (LCM) analog Le Fort 1; c Frakturmuster b + Fraktur in Jochbogenmitte, Involvierung von Stirnbeinpfeiler und Orbitadach und medialer Orbitawand ( $\Sigma$ Orbita-4-Wand-Fraktur), Multifragmentierung von LCM, ZMC und Zygomatic orbital Flange.

formation - wie in 3-D-Spielen - eingesetzt werden müssten.

Im Moment kommt es in erster Linie aber darauf an, der CMF-Klassifikation z.B. im Rahmen von Multicenterstudien, zu allgemeiner Verbreitung zu verhelfen, um einen Anfang zu machen, Expertise aufzubauen und langfristig Konventionen zur Eingruppierung zu schaffen. Erfahrugsgemäß geht das nicht ohne Instruktionen und Training für den Anwenderkreis, sowie eine komfortable Verlinkung mit Klinik-Dokumentationsund -Vergütungssystemen. Weiterentwicklungen und Verbesserungen werden sich v.a. anhand nicht eindeutiger oder sog. komplexer Fälle ergeben.

Die Ausdrucksweise „komplexe“ Frakturen ist indessen wenig hilfreich, solange diese nicht in Bezug auf Lokalisation, assoziierte Subregionen und morphologische Eigenschaften definiert sind. Spaßeshalber wird im Klinikalltag von „Le Fort IV“-Frakturen gesprochen, wofür 
sich jeder in seiner Fantasie andere Umrisse ausmalt.

Die Vorstellungen und Wünsche für zukünftige Entwicklungen reichen von der Kompatibilität mit Apple/Mac-Betriebssystemen, über Touchscreen/Tablet-Applikationen bis zu automatischer Bildanalyse und Klassifikation über Fusionierung von CT-Daten und Klassifizierungs-Templates inkl. Volumenbestimmungen der Orbitae (Strong et al. 2013).

Jeder technologische Fortschritt in Bildgebung und Evalutionsmethodik wird jedoch nicht nur zu Verbesserungen bei Befunderhebung und Diagnosefindung beitragen, sondern auch die Unzulänglichkeiten bestehender Klassifikationssysteme offenlegen.

Abschließend geben die Autoren ihrer Hoffnung Ausdruck, dass die 3. AO CMF Fracture Classification den Kern fortgesetzter Aktivitäten bei der Erarbeitung einer allgemein akzeptierten Fraktureneinteilung bilden möge.

Und ganz zum Schluss sei kurz an den Sinn und Zweck einer Frakturenklassifikation erinnert: die Klassierung ist als Wegweiser gedacht, ein individuelles Mapping der notwendige Folgeschritt als Grundlage zur Behandlung.

\section{Widmung}

Der Beitrag ist Herrn Prof. Dr. Wolfgang Dauber, Tübingen - Anatomielehrer des Erstautors - zum 75. Geburtstag gewidmet!

\section{Anmerkungen}

- Die Nomenklatur der AOCMF Fracture Classification ist in den Abbildungen in englischer Sprache belassen.

- Ein Skull Base/Cranial Vault Module (Di leva et al. 2013) wird hier nicht ausgeführt.

- Ab Anfang 2014 wird über Links auf der Website der AO Foundation ein Download der neuesten Windows AOCOIAC-Version mit der vollständigen AO-CMF-Frakturenklassifikation zur Verfügung stehen.

\section{Literatur}

${ }^{1}$ Audigé L, Bhandari M, Hanson B et al. A concept for the validation of fracture classifications. J Orthop Trauma 2005; 19: 401-406

2 Audigé L, Cornelius CP, Di Ieva A et al.; CMF Classification Group 2013. Introduction to the Comprehensive AO Classification System for fractures of the craniomaxillofacial skeleton: rationale, methodological background, developmental process and objectives. Craniomaxillofac Trauma Reconstr (in press)

${ }^{3}$ Audigé L, Cornelius CP, Buitrago-Téllez CH et al. The Comprehensive AO Classification system for fractures of the craniomaxillofaxial skeleton: software solution for documentation. Craniomaxillofac Trauma Reconstr (in press)

${ }^{4}$ Bächli H, Leiggener C, Gawelin P et al. Skull base and maxillofacial fractures: Two centre study with correlation of clinical findings with a comprehensive craniofacial classification system. J Craniomaxillofac Surg 2009; 37: 305-311

${ }^{5}$ Bagheri SC, Dierks EJ, Kademani D et al. Application of a facial injury severity scale in craniomaxillofacial trauma. J Oral Maxillofac Surg 2006; 64: 408-414

${ }^{6}$ Buitrago-Téllez CH. Neue therapiebezogene Klassifikation von Mittelgesichtsfrakturen Postmortale experimentelle und klinische Studien mit 2- und 3-dimensionaler Computertomographie. Freiburg: Habilitationsschrift Albert Ludwigs-Universität; 1998

${ }^{7}$ Buitrago-Téllez CH, Schilli W, Bohnert M et al. A comprehensive classification of craniofacial fractures: postmortem and clinical studies with two- and three-dimensional computed tomography. Radiology 1999; 213 (Suppl.): S445

${ }^{8}$ Buitrago-Téllez $\mathrm{CH}$, Schilli W, Bohnert $M$ et al. A comprehensive classification of craniofacial fractures: postmortem and clinical studies with two- and three-dimensional computed tomography. Injury 2002; 33: 651-668

${ }^{9}$ Buitrago-Téllez $\mathrm{CH}$, Audigé L, Strong B et al. A comprehensive classification of mandibular fractures: a preliminary agreement validation study. Int J Oral Maxillofac Surg 2008: 37: 1080-1088

10 Buitrago-Téllez CH, Audigé L, Cornelius CP et al. Systematic radiological approach for diagnosis and classification of craniomaxillofacial fractures using the Comprehensive AO System. Craniomaxillofac Trauma Reconstr (in press)

11 Carinci F, Zollino I, Brunelli G et al. Orbital fractures: a new classification and staging of 190 patients. J Craniofac Surg 2006: 17: 1040-1044

12 Chen CH, Wang TY, Tsay PK et al. A 162-case review of palatal fracture: management strategy from a 10 -year experience. Plast Reconstr Surg 2008; 121: 2065-2073

13 Clark N, Birely B, Manson PN et al. High-energy ballistic and avulsive facial injuries: classification, patterns, and an algorithm for primary reconstruction. Plastic Reconstr Surg 1996; 98 (Suppl. 1): 583-601

14 Cooter RD, David DJ. Computer-based coding of fractures in the craniofacial region. $\mathrm{Br} \mathrm{J}$ Plast Surg 1989; 42: 17-26

15 Cornelius CP, Audigé L, Prein J. AO Classification of craniomaxillofacial Fractures. In: Ehrenfeld M, Manson PN, Prein J, eds. Principles of internal Fixation of the craniomaxillofacial Skeleton-Trauma and orthognathic Surgery. Stuttgart, New York: Georg Thieme Verlag; 2012: 9-14

16 Cornelius CP, Audigé L, Kunz C et al. The Comprehensive AO Classification System for fractures of the craniomaxillofaxial skeleton: Mandible fractures Level 2. Craniomaxillofac Trauma Reconstr (in press)

17 Cornelius CP, Audigé L, Kunz C et al. The Comprehensive AO Classification System for fractures of the craniomaxillofaxial skeleton: Mandible fractures Level 3. Craniomaxillofac Trauma Reconstr (in press)
18 Cornelius CP, Audigé L, Kunz C et al. The comprehensive AO Classification System for fractures of the craniomaxillofaxial skeleton: Midface fractures Level 3. Craniomaxillofac Trauma Reconstr (in press)

${ }^{19}$ Cornelius CP, Kunz C, Audigé L et al. The Comprehensive AO Classification System for fractures of the craniomaxillofaxial skeleton: Mandible and Midface Fractures - Case Collection. Journal Maxillofacial Trauma \& Reconstruction (in press)

20 Cornelius CP, Probst FA, Ratgeber JPC et al. Zur Osteosynthese bei Frakturen im zahnlosen atrophen Unterkiefer - Möglichkeiten zum Hardware Downsizing, Lingual Plating. OPJournal 2013; 29: 164-174

${ }^{21}$ Di leva A, Audigé L, Kellman RM et al. The Comprehensive AO Classification System for fractures of the craniomaxillofaxial skeleton: Skull base and cranial vault fractures Level 2 and 3. Craniomaxillofac Trauma Reconstr (in press)

22 Donat TL, Endress C, Mathog RH. Facial fracture classification according to skeletal support mechanisms. Arch Otolaryngol Head Neck Surg 1998; 124: 1306-1314

${ }^{23}$ Gruss JS, Hurwitz IJ, Nik NA, et al. The pattern and incidence of nasolacrimal injury in nasoorbital-ethmoid fractures: the role of delayed assessment and dacryocystorhinostomy. $\mathrm{Br} J$ Plast Surg 1985; 38: 116-121

${ }^{24}$ Hammer B. Orbital Fractures Diagnosis, operative Treatment, secondary Correction. Seattle, Toronto, Bern: Hogrefe und Huber; 1995

25 Jackson IT. Classification and treatment of orbito-zygomatic and orbitoethmoid fractures - the place of bone grafting and plate fixation. Clin Plast Surg 1989; 16: 77-91

${ }^{26}$ Jaquiéry C, Äeppli P, Cornelius CP. Reconstruction of orbital wall defects: critical review of 72 patients. Int J Oral Maxillofac Surg 2007; 36: 193-199

${ }^{27}$ Jaquiéry C Leiggener C, Kunz C et al. Aktuelle Behandlungsstrategien von knöchernen Verletzungen der Orbita. OP-Journal 2013; 29: 136-146

28 Joos U, Meyer U, Tkotz T et al. Use of a mandibular fracture score to predict the development of complications. J Oral Maxillofac Surg 1999; 57: 2-5

${ }^{29}$ Kunz C, Cornelius CP, Prein J et al. The Comprehensive AO Classification System for fractures of the craniomaxillofaxial skeleton: Midface fractures - Level 2. Craniomaxillofac Trauma Reconstr (in press)

${ }^{30}$ Kunz C, Cornelius CP, Audigé L et al. The Comprehensive AO Classification System for fractures of the craniomaxillofaxial skeleton: Orbital fractures - Level 3. Craniomaxillofac Trauma Reconstr (in press)

${ }^{31}$ Le Fort $R$. Étude Experimentale Sur Les Fractures De La Machoire Supérieure, Part I, Part II, Part III. Paris: Rev Chir 1901; 23: 208-227, 360-379, 479-507

32 Loukota RA, Eckelt $U$, De Bont $L$ et al. Subclassification of fractures of the condylar process of the mandible. $\mathrm{Br}$ J Oral Maxillofac Surg 2005: 43: 72-73

${ }^{33}$ Luhr G, Reidick T, Merten HA. Frakturen des atrophischen Unterkiefers - eine Herausforderung für die Therapie. Fortschr Kiefer Gesichtschir 1996; 41: 151-154

34 Luhr HG, ReidickT, Merten HA. Results of treatment of fractures of the atrophic edentulous mandible by compression plating: a retrospective evaluation of 84 consecutive cases. J Oral Maxillofac Surg 1996; 54: 250-254

35 Madhusudan G, Sharma RK, Khandelwal N et al. Nomenclature of frontobasal trauma: a new clinicoradiographic classification. Plast Reconstr Surg 2006; 117: 2382-2388 
36 Manson PN. Some thoughts on the classification and treatment of Le Fort fractures. Ann Plast Surg 1986; 17: 356-363

37 Markowitz BL, Manson PN, Sargent L et al. Management of the medial canthal tendon in nasoethmoid orbital fractures: the importance of the central fragment in classification and treatment. Plast Reconstr Surg 1991; 87: 843-853

38 Müller M, Narzarian S, Koch P et al. The comprehensive Classification for Fractures of long Bones. Berlin, Heidelberg, New York: Springer; 1990

39 Neff A, Audigé L, Rasse $M$ et al. The Comprehensive AO Classification System for fractures of the craniomaxillofaxial skeleton: Mandibular condylar process fractures - Level 3. Craniomaxillofac Trauma Reconstr (in press)

40 Nolasco FP, Mathog RH. Medial orbital wall fractures: classification and clinical profile. Otolaryngol Head Neck Surg 1995; 112: 549-556

41 Rafferty MA, Mc Conn Walsh R, Walsh MA. A comparison of temporal bone fracture classification systems. Clin Otolaryngol 2006; 31 : 287-291

42 Roth FS, Kokoska MS, Awwad EE et al. The identification of mandible fractures by helica computed tomography and panorex tomography. J Craniofac Surg 2005; 16: 394-399

43 Schiel S, Smolka W, Leiggener C et al. „Open book“-Frakturen des Mandibularbogens: bilaterale Gelenkfortsatzfrakturen in Kombination mit Paramedian-/Medianfrakturen des Unterkiefers. Operative Behandlungsstrategien. OP-Journal 2012; 28: 194-210

44 Schiel S, Probst FA, Mayer P et al. Kiefergelenkfortsatzbasis- und Kiefergelenkhalsfrakturen bei Kindern und Jugendlichen-Plädoyer für eine transoral endoskopisch assistierte operative Versorgung bei starker Dislokation und Luxation. OP-Journal 2013; 29: 156-163

45 Schmalzriedt E. Inhumane Klassik, Vorlesung wider ein Bildungsklischee mit den wichtigsten Dokumenten zur Tradition eines fragwürdigen Begriffs. München: Kindler; 1971

46 Slongo T, Audigé L; AO Pediatric Classification Group. Fracture and dislocation classification compendium for children - The AO pediatric comprenhensive classification of long bone fractures (PCCF). J Orthop Trauma 2007; 21 (Suppl. 10): S135-S160

47 Spiessl B, Schroll K. Gelenkfortsatz- und Gelenkköpfchenfrakturen. In: Nigst $\mathrm{H}$, Hrsg. Spezielle Frakturen- und Luxationslehre, Band I/1 Gesichtsschädel. Stuttgart, New York: Georg Thieme Verlag; 1972: 136-152

48 Spiessl B. Internal Fixation of the Mandible. A Manual of AO/ASIF Principles. Heidelberg, New York: Springer; 1989

49 Strong EB, Fuller SC, Chahal SH. ComputerAided Analysis of Orbital Volume: A Novel Technique. Ophthal Plast Reconstr Surg 2013; 29: 1-5

${ }^{50}$ Tessier $P$. The classic reprint. Experimental study of fractures of the upper jaw. Parts and II. In: Le Fort R, Lille MD (Rev. chir. de Paris 23, 208-227, 360-379, 1901). Paris: Plast Reconstr Surg 1972; 50: 497-506
51 Tessier $P$. The classic reprint. Experimental study of fractures of the upper jaw. Part III. In: Le Fort R, Lille MD (Rev. chir. de Paris 23, 479-507, 1901). Paris: Plast Reconstr Surg 1972: 50: 600-605

52 Wassmund M. Frakturen und Luxationen des Gesichtsschädels. Berlin: Meusser 1927

53 Zhang J, Zhang Y, El-Maaytah M et al. Maxillofacial Injury Severity Score: proposal of a new scoring system. Int J Oral Maxillofac Surg 2006 35: 109-114

54 Zingg M, Laedrach $K$, Chen $J$ et al. Classification and treatment of zygomatic fractures: a review of 1025 cases. J Oral Maxillofac Surg 1992; 50: 778-790

Prof. Dr. med. Dr. med. dent.

\section{P. Cornelius}

Oberarzt der Klinik

Klinik und Poliklinik für Mund-,

Kiefer- und Gesichtschirurgie

Klinikum der Universität München

Ludwig-Maximilians-Universität

München

Lindwurmstr. 2a

80337 München

Peter.Cornelius@med-uni.muenchen.de

Priv.-Doz. Dr. med vet.

Laurent Audigé, PhD

Senior Research Fellow -

Upper Extremities

Schulthess Klinik

Muskuloskelettal Zentrum

Lengghalde 2

8008 Zürich

Schweiz

AO Clinical Investigation

and Documentation

Stettbacherstr. 6

8600 Dübendorf

Schweiz

Priv.-Doz. Dr. med. Dr. med. dent.

\section{Christoph Kunz}

Stellvertretender Chefarzt,

Leitender Arzt

Mund-, Kiefer- und Gesichtschirurgie

Universitätsspital Basel

Spitalstr. 21

4031 Basel

Schweiz
Prof. Dr. med. C. H. Buitrago-Téllez

Chefarzt Radiologie

Kantonsspital Zofingen

Mühlethalstr. 27

4800 Zofingen

Schweiz

Prof. Dr. med. Dr. med. dent.

Andreas Neff

Direktor der Klinik

Klinik für Mund-Kiefer-

Gesichtschirurgie

Universitätsklinikum Marburg

Zentrum für ZMK-Heilkunde

Georg-Voigt-Str. 3

35039 Marburg

Prof. Dr. med. Dr. med. dent. Michael Rasse

Direktor der Klinik für Mund-, Kiefer- und Gesichtschirurgie Geschäftsführender Direktor

Department für Zahn,- Mund- und Kieferheilkunde und Mund-, Kieferund Gesichtschirurgie Universitätsklinik Innsbruck

Anichstr. 35

6020 Innsbruck

Österreich

Prof. Dr. med. Dr. med. dent. Joachim Prein

AO Foundation Office Dübendorf

Stettbacherstr. 6

8600 Dübendorf

Schweiz

Direktor em.

Wiederherstellungschirurgie, Mund-, Kiefer- und Gesichtschirurgie Universitätsspital Basel 